OPEN ACCESS

Edited by:

Giamila Fantuzzi,

University of Illinois at Chicago,

United States

Reviewed by:

John Kelley Bentley,

The University of Michigan Medical

School, Ann Arbor, United States

Katarzyna Kaczynska,

Mossakowski Medical Research

Centre (PAN), Poland

*Correspondence:

Rupali Das

dasrupal@msu.edu

Specialty section:

This article was submitted to

Inflammation,

a section of the journal

Frontiers in Immunology

Received: 30 September 2021

Accepted: 18 October 2021

Published: 29 October 2021

Citation:

Subramanian H, Hashem T, Bahal D

Kammala AK, Thaxton $K$ and Das $R$

(2021) Ruxolitinib Ameliorates

Airway Hyperresponsiveness

and Lung Inflammation in a

Corticosteroid-Resistant Murine

Model of Severe Asthma.

Front. Immunol. 12:786238.

doi: 10.3389/fimmu.2021.786238

\section{Ruxolitinib Ameliorates Airway Hyperresponsiveness and Lung Inflammation in a Corticosteroid- Resistant Murine Model of Severe Asthma}

\author{
Hariharan Subramanian ${ }^{1}$, Tanwir Hashem ${ }^{2}$, Devika Bahal ${ }^{3}$, Ananth K. Kammala ${ }^{1}$, \\ Kanedra Thaxton ${ }^{2}$ and Rupali Das ${ }^{1 *}$ \\ 1 Department of Physiology, College of Human Medicine, Michigan State University, East Lansing, MI, United States, \\ ${ }^{2}$ College of Natural Science, Michigan State University, East Lansing, MI, United States, ${ }^{3}$ College of Veterinary Medicine, \\ Michigan State University, East Lansing, MI, United States
}

Asthma prevalence has increased considerably over the decades and it is now considered as one of the most common chronic disorders in the world. While the current antiasthmatic therapies are effective for most asthma patients, there are 5-10\% subjects whose disease is not controlled by such agents and they account for about $50 \%$ of the asthma-associated healthcare costs. Such patients develop severe asthma (SA), a condition characterized by a dominant Th1/Th17 cytokine response that is accompanied by Type 2 (T2)-low endotype. As JAK (Janus Kinase) signaling is very important for the activation of several cytokine pathways, we examined whether inhibition of JAKs might lessen the clinical and laboratory manifestations of SA. To that end, we employed a recently described murine model that recapitulates the complex immune response identified in the airways of human SA patients. To induce SA, mice were sensitized with house dust mite extract (HDME) and cyclic (c)-di-GMP and then subsequently challenged with HDME and a lower dose of c-di-GMP. In this model, treatment with the JAK inhibitor, Ruxolitinib, significantly ameliorated all the features of SA, including airway hyperresponsiveness and lung inflammation as well as total lgE antibody titers. Thus, these studies highlight JAKs as critical targets for mitigating the hyperinflammation that occurs in SA and provide the framework for their incorporation into future clinical trials for patients that have severe or difficult-to manage asthma.

Keywords: severe asthma, corticosteroid resistance, T2-low asthma, ruxolitinib, house dust mite extract (HDME), airway hyperresponsiveness (AHR), lung inflammation, interleukin (IL)-17 


\section{INTRODUCTION}

Asthma affects more than 300 million people worldwide and is associated with significant morbidity and mortality $(1,2)$. It is deemed as a major public health issue as the direct medical expense of asthma treatment and the indirect costs associated with time lost from work and premature death contribute to the increased global economic burden (2). Clinically, asthma is a chronic inflammatory disease often characterized by recurrent episodes of wheezing, coughing, shortness of breath and chest tightness that is caused due to airway hyperresponsiveness (AHR), bronchoconstriction, increased mucus production and airway remodeling $(1,2)$.

Traditionally, asthma is defined as two distinct forms: nonallergic and allergic phenotypes, the latter being the most common form of asthma and is caused by sensitization to allergens such as pollen, mold, cockroach, pet dander, and dust mites (1). However, it is now well accepted that asthma is a complex heterogenous disease that is regulated by various inflammatory pathways $(1,2)$. Thus, based on the pathophysiologic mechanisms, asthma is now classified as Type 2 (T2)-high and T2-low endotypes, as depicted in Figure 1A. In T2-high asthma, CD4+T helper 2 (Th2) cells and innate lymphoid cells group 2 (ILC2), eosinophils, immunoglobulin $\mathrm{E}$ (IgE) and T2 cytokines such as interleukin (IL)-4, IL-5 and IL-13 contribute to its pathogenesis. In contrast, the pathobiology of T2low asthma typically involves Th1 and Th17 cells, neutrophils and several proinflammatory cytokines such as IL-1 $\beta$, IL-6, IL-17A, IL$17 F$, IFN- $\gamma$ and TNF- $\alpha$ (2).

The current anti-inflammatory drugs for asthma treatment include corticosteroids (CS) and anti-leukotrienes, that are

A
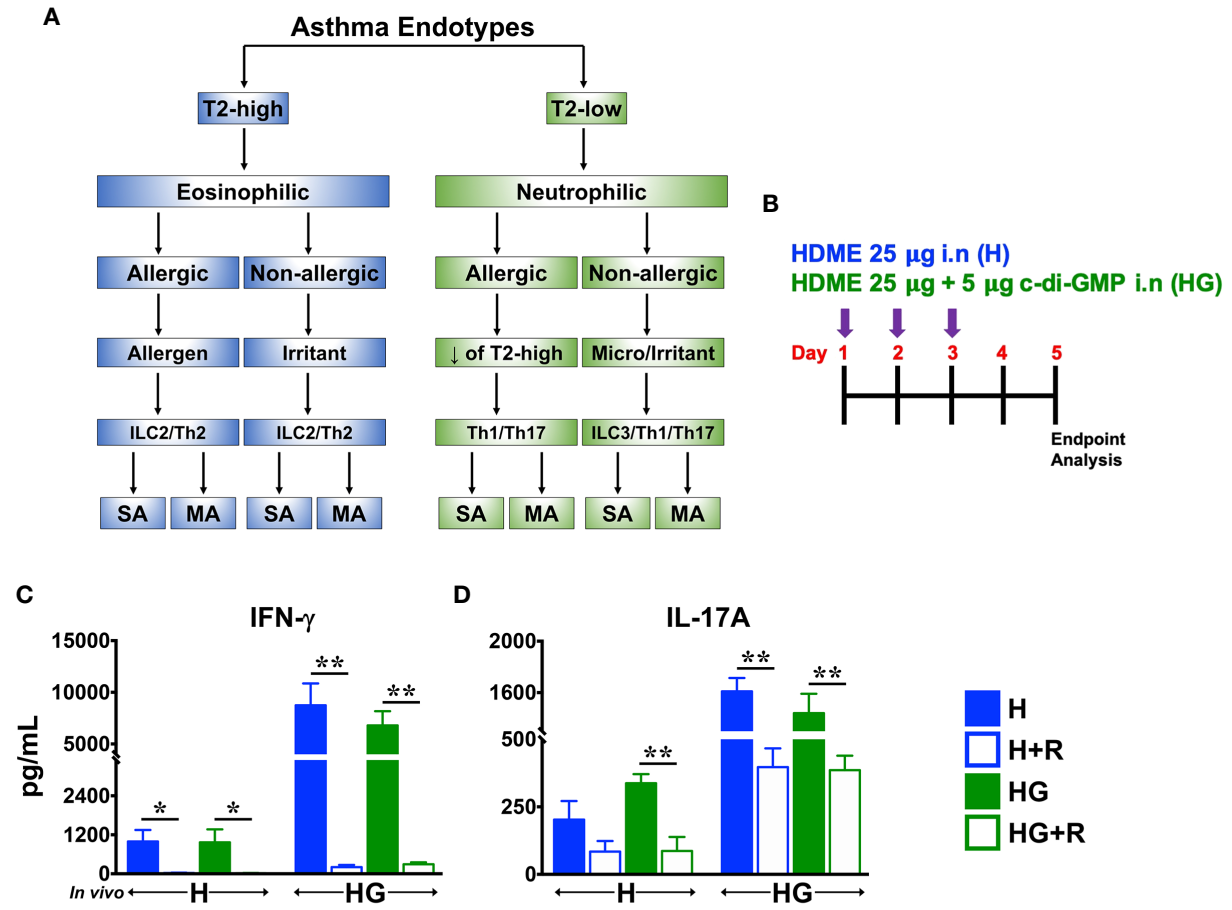

IL-17A
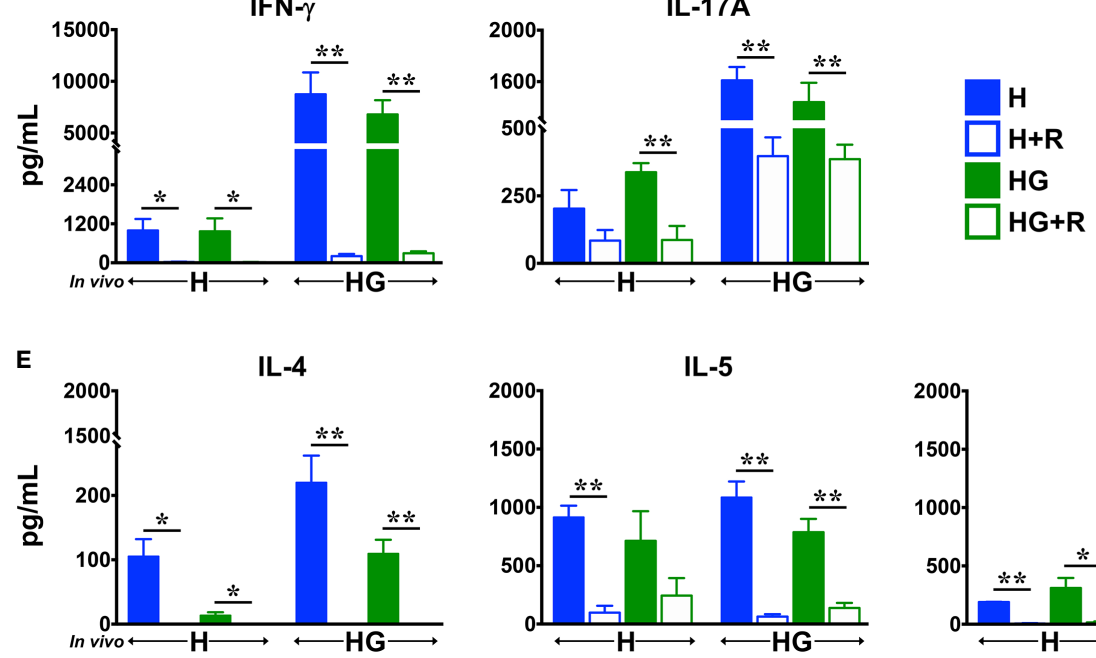

FIGURE 1 | Ruxolitinib reduces HDME- and HDME+C-di-GMP-induced Th1, Th2/T2 and Th17 cytokine production in vitro. (A) Classification of asthma endotypes. (B) Schematics of short-term in vivo sensitization. Balb/c mice were challenged intranasally (i.n) with HDME $(25 \mu \mathrm{g})$ or HDME $(25 \mu \mathrm{g})+\mathrm{C}-\mathrm{di}-\mathrm{GMP}(5 \mu \mathrm{g})$ for 3 consecutive days. Forty-eight hours after the last injection, mice were sacrificed and lungs were harvested for cytokine analysis. (C-E) Lung mononuclear cells were isolated and cultured ex vivo in the presence of either HDME $(H)$ or HMDE+c-di-GMP $(\mathrm{HG})$, with or without Ruxolitinib (R), as indicated. After 72 hours, cell culture supernatants were analyzed for (C) IFN- $\gamma$, (D) IL-17A, and (E) T2 cytokines (IL-4, IL-5 and IL-13). Data is shown as mean \pm SEM and pooled from 3 independent experiments with a total of 3-9 mice per cohort. Statistical significance was determined by Student's unpaired $t$ test with Welch's correction. ${ }^{* *} p \leq 0.01,{ }^{*} p \leq 0.05$. $\mathrm{SA}$, severe asthma; MA, mild asthma; micro, microbes. 
effective for most of asthma patients (2). However, there are about $5-10 \%$ subjects that develop severe asthma (SA) and do not respond to these agents (1). Such severe asthmatics require frequent hospitalizations and/or need emergency care, contributing up to $50 \%$ of health costs associated with asthma (1). In a prior study (3), Raundhal et al. reported that SA patients have a dominant Th1 immune response inspite of ongoing treatment with high doses of CS, highlighting the need for the development of newer and effective therapies.

Most pro-inflammatory cytokines signal through Janus Kinase (JAK) proteins (4). JAKs are a family of four tyrosine kinases (JAK1, JAK2, JAK3 and Tyk2) that selectively associate with cytokine receptor chains and mediate signaling by phosphorylating tyrosine residues on themselves, the cytokine receptor chains and STAT (signal transducer and activator of transcription) proteins (4). JAK1 plays a major role in the signaling of several proinflammatory cytokines, often in association with other JAK family members, such as JAK2 or JAK3 $(4,5)$. A number of JAK inhibitors have been developed for clinical use in inflammatory diseases, including asthma $(5,6)$. However, the effect of JAK inhibitors on the immunopathology of CS-resistant SA remains to be investigated.

In the current study, we examined the effect of Ruxolitinib (7), a potent inhibitor of JAK1/2, on the pathogenesis of CS-resistant SA. To that end, we used a recently developed murine model of SA that recapitulates the immune pathophysiology of severe asthmatics unresponsive to CS (3). Consistent with a prior report (3), we observed that intranasal administration of HDME and cdi-GMP (a mucosal adjuvant as well as a potent STING [Stimulator of Interferon genes] agonist) induced AHR and lung inflammation in mice. These SA mice exhibited high serum IgE levels and had significantly increased numbers of both eosinophils and neutrophils in their lungs. Consistent with the mixed granulocytic infiltration, lungs of SA mice expressed enhanced gene transcripts of chemokines and cytokines that drive eosinophilic and neutrophilic inflammation. Importantly, Ruxolitinib significantly reduced $\mathrm{HDME}+\mathrm{c}-\mathrm{di}-\mathrm{GMP}$-mediated AHR, lung inflammation and serum IgE levels. However, this amelioration in the SA features was associated with suppression of cytokines and chemokines that predominantly regulate Th1 and T2 immune response, independent of the cellular factors that regulate neutrophil recruitment and function. Lastly, we demonstrate that Ruxolitinib critically modulates expression of several microRNAs that have known roles in the pathogenesis of SA.

\section{MATERIALS AND METHODS}

\section{Mice}

Balb/c mice were purchased from Jackson Laboratories (Bar Harbor, ME); bred and housed under specific pathogen-free conditions. Female mice (8-10 weeks old) were used for experiments. All animal studies were approved by the Institutional Animal Care and Use Committee at the Michigan State University (protocol number: PROTO202000162).

\section{Mouse Model of Severe Asthma}

A previously described mouse model of severe asthma was used (3). Age-matched Balb/c female mice were intranasally (i.n) sensitized using $25 \mu \mathrm{g}$ of house dust mite extract (HDME) $(1 \mu \mathrm{g} / \mu \mathrm{L}, 28750 \mathrm{EU} / \mathrm{vial}$; Stallergenes Greer, UK) mixed with $5 \mu \mathrm{g}$ of c-di-GMP (Invivogen, San Diego, CA) on days 1, 3 and 5. After 5 days of rest, mice were re-challenged with HDME $(25 \mu \mathrm{g})$ and a lower dose of c-di-GMP $(0.5 \mu \mathrm{g})$ on day 11 and with HDME only $(25 \mu \mathrm{g})$ on days 12 and 13 . Mice were similarly rechallenged two more times on days $18-20$ and $25-27$. For all i.n administrations, mice were anasthesized with $2.5 \%$ isofluorane. HDME was reconstituted in sterile PBS at a concentration of $2 \mu \mathrm{g}$ of protein $/ \mu \mathrm{L}$. The amount of HDME protein, Derp1 and endotoxin was $50 \mu \mathrm{g}, 3.1 \mu \mathrm{g}$ and $38.4 \mathrm{EU}$ respectively per injection in a volume of $25 \mu \mathrm{L}$. Ruxolitinib (LC Laboratories, Woburn, MA) was prepared in citrate buffer ( $\mathrm{pH} 3.5$ ) with $20 \%$ (wt/vol) Captisol (Ligand Pharmaceuticals, La Jolla, CA) and was given i.n $(10 \mathrm{mg} / \mathrm{kg}$ body weight) starting on the first day of the challenge for 2 consecutive days followed by a day of rest for a total of 12 injections. Schematic of this model is shown in Figure 2A. Twenty-four hours after the final HDME challenge, mice were anesthetized for measurement of airway hyperresponsiveness (AHR) and then sacrificed for collection of blood, bronchoalveolar lavage fluid (BALF) and lung tissue for various endpoint analysis.

\section{AHR Assessment}

Mice were anesthetized using $100 \mathrm{mg} / \mathrm{kg}$ ketamine (Henry Schein Animal Health, Dublin, OH), $10 \mathrm{mg} / \mathrm{kg}$ xylazine (Akorn, Lake Forest, IL) and $3 \mathrm{mg} / \mathrm{kg}$ acepromazine (Henry Schein Animal Health, Dublin, $\mathrm{OH}$ ) through intraperitoneal (i.p) injection. Subsequently mice were tracheostomized and airway mechanics was measured using flexiVent (SCIREQ ${ }^{\circledR}$, Quebec, Canada). Assessment of AHR such as central airway resistance [Newtonian resistance $(\mathrm{Rn})$ ] was measured by a methacholine (MCh; Sigma-Aldrich, St. Louis, MO) challenge test with increasing doses of $\mathrm{MCh}(0-100 \mathrm{mg} / \mathrm{ml}$, as indicated in Figure 2B) in $100 \mu \mathrm{L}$ volume, using a nebulizer.

\section{Serum and Bronchoalveolar Lavage Fluid (BALF) Collection}

Blood was collected from the superior mesenteric vein of the mouse and left at $4^{\circ} \mathrm{C}$ overnight. Serum was collected the next day and analyzed for total IgE and IgG1 using commercially available ELISA kits from Invitrogen (Carlsbad, CA). For BALF collection, mice were euthanized and whole lung was lavaged with $0.6 \mathrm{ml}$ of sterile PBS three times. The resultant BALF was centrifuged to separate the cellular components from the supernatants. Total BALF cellularity was determined using a hemacytometer.

\section{Lung Histology}

The lungs were infused via the trachea with $10 \%$ buffered formalin. After excision, the lungs were immersed in fresh $10 \%$ formalin overnight. Samples were then embedded in paraffin, cut into $5-\mu \mathrm{m}$-thick sections and stained with hematoxylin and eosin 
(H\&E). Digital images of sections were obtained using a Nikon Eclipse 50i microscope (Nikon, Japan) equipped with a INFINITY-3 digital color camera (Lumenera Corporation, Canada), and INFINITY ANALYZE 6.5.4 software.

\section{Quantitative Real-Time PCR}

Lungs were dissociated in TRIzol solution (Thermo Fisher Scientific, Waltham, MA) using a high-speed homogenizer (Fisher Scientific, Hampton, NH) and total RNA was extracted as per manufacturer's protocol. RNA $(2 \mu \mathrm{g})$ was reverse transcribed into cDNA using SuperScript III in a $20 \mu \mathrm{l}$ reaction volume or using the Taqman Advanced miRNA cDNA synthesis kit (for microRNA analysis) according to the manufacturer's instructions (Applied Biosystems, Foster City, CA). Real-time quantitative PCR was performed using Quant Studio $^{\text {TM }} 3$ system (Applied Biosystems) with validated Taqman primers and Fast Advanced Master Mix according to manufacturer's instructions. Relative gene expression data (fold change) between samples was accomplished using the $2^{-\Delta \Delta \mathrm{Ct}}$ method. GAPDH (for gene expression) or $18 \mathrm{~S}$ (for miRNA analysis) was used as the internal reference control.

\section{Isolation of Immune Cell Populations From the Lungs}

Lung samples were digested with collagenase P $(1 \mathrm{mg} / \mathrm{ml}$, Roche Diagnostics, Indianapolis, IN) at $37^{\circ} \mathrm{C}$ for 30 minutes. Single cell suspension was obtained by passing the digested tissue through a $70 \mu \mathrm{m}$ cell strainer (Alkali Scientific Inc, Fort Lauderdale, FL) with a plunger. Lung immune cells were then isolated using density centrifugation with Percoll (GE, Piscataway, NJ). The isolated cells were washed and resuspended in RPMI media (Life Technologies, Carlsbad, CA) conditioned with 10\% FBS (Atlanta Biologicals, Flowery Branch, GA) and $1 \%$ penicillinstreptomycin (Mediatech Inc, Manassas,VA). Total lung cellularity was determined using a hemacytometer.

\section{Re-Stimulation of Lung Immune Cells In Vitro}

Lung immune cells $(0.2 \times 10$ (6) cells/well) were plated with either HDME $(3 \mu \mathrm{g} /$ well $)$ or HDME $(3 \mu \mathrm{g} /$ well $)+$ c-di-GMP $(0.5$ $\mu \mathrm{g} /$ well $)$ without or with Ruxolitinib $(1 \mu \mathrm{M} /$ well $)$ in triplicates and incubated at $37^{\circ} \mathrm{C}$. After 72 hours, culture supernatant was collected and levels of IFN- $\gamma$, IL-17A, IL-4, IL-5 and IL-13 were measured using ELISA kits (Invitrogen and BD Bioscience).

\section{Antibodies and Flow Cytometry}

The antibodies used for immunofluorescence staining include CD4, CD11b, B220, DX5, TCR $\beta$, and SiglecF (BD Biosciences). CD8a, CD11c, IA/IE (MHCII), and Ly6G were from Biolegend, San Diego, CA. Fluorochrome conjugated CD1d-tetramer (CD1d-Tet) loaded with glycolipid antigen (PBS57), or unloaded controls were provided by the NIH Tetramer Core Facility (Emory University, Atlanta, GA). Data was collected on a LSRII flow cytometer (BD Biosciences) and analyzed using FlowJo software (FlowJo LLC, Ashland, OR).

\section{Statistics}

Statistical significance was determined using GraphPad Prism software (GraphPad, San Diego, CA). Student's $t$-test with Welch's correction or two-way ANOVA with Tukey's multiple comparisons test were used as indicated in the figure legends. Significance is shown as ${ }^{\star}(\mathrm{p}<0.05),{ }^{* *}(\mathrm{p}<0.01)$, or ns for nonsignificant values.

\section{RESULTS}

\section{Ruxolitinib Suppresses HDME- and HDME+c-di-GMP-Induced Cytokine Production In Vitro}

Type 2 (T2)-high asthma is associated with elevated levels of Th2 cytokines such as IL-4, IL-5 and IL-13 whereas T2-low asthma is mainly linked to the activation of Th1 and Th17 cells (2) (Figure 1A). Others (8) and we (9) have previously shown that repeated intransal exposure to HDME (a naturally inhaled antigen) results in a T2-polarized eosinophilic lung inflammation and airway hyperresponsiveness (AHR) in mice, similar to that seen in human asthmatics. In contrast, the second messenger c-di-GMP induces a robust Th1/Th17 but a modest T2 response (3). To investigate the effect of Ruxolitinib on allergen-induced cytokine production, we sensitized wild-type $\mathrm{Balb} / \mathrm{c}$ mice with repeated high doses of $\operatorname{HDME}(\mathrm{H})$ or HDME+c-di-GMP (HG) (Figure 1B). We observed that lung immune cells from mice sensitized with HG produced significantly higher levels of IFN- $\gamma$ and IL-17A (hallmark cytokines of Th1 and Th17 cells, respectively) when stimulated with either HDME or HG in vitro, as compared to HDMEtreated mice (Figures 1C, D). However, comparable amounts of T2 cytokines (with the exception of IL-13) were produced by lung cells from mice sensitized with either HDME or $\mathrm{HG}$ (Figure 1E). Strikingly, when lung immune cells from these animals were stimulated with either HDME or HG in presence of Ruxolitinib in vitro; levels of all the cytokines in culture supernatants were significantly reduced (Figures 1C-E).

\section{Ruxolitinib Attenuates AHR, IgE Production and Lung Inflammation in SA Mice}

In light of the in vitro findings (Figure 1), we next determined the effect of Ruxolitinib in vivo, using a murine model that recapitulates the corticosteroid (CS)-refractory immune response seen in severe human asthmatics (Figure $\mathbf{2 A}$ ). In this model (3), intranasal (i.n) injection of HG induces a mixed Th1 and Th17 immune response that is accompanied with a low T2 activity. As depicted in Figure 2A, we administered Ruxolitinib i.n in the challenge phase; once a day for two consecutive days. After a day of rest, the drug treatment was repeated until the end of the study for a total of 6 treatment sets (Figure 2A). PBScontrol and severe asthma (SA: HG-treated) mice without or with Ruxolitinib $(\mathrm{SA}+\mathrm{R})$ were anesthetized and AHR [central airway resistance $(\mathrm{Rn})]$ was assessed in response to methacholine (Mch) challenge. Compared to PBS controls, SA mice 
A

HDME $25 \mu \mathrm{g}+5 \mu \mathrm{g}$ di-CGMP i.n

HDME $25 \mu \mathrm{g}+0.5 \mu \mathrm{g}$ di-CGMP i.n

HDME $25 \mu \mathrm{g}$ i.n
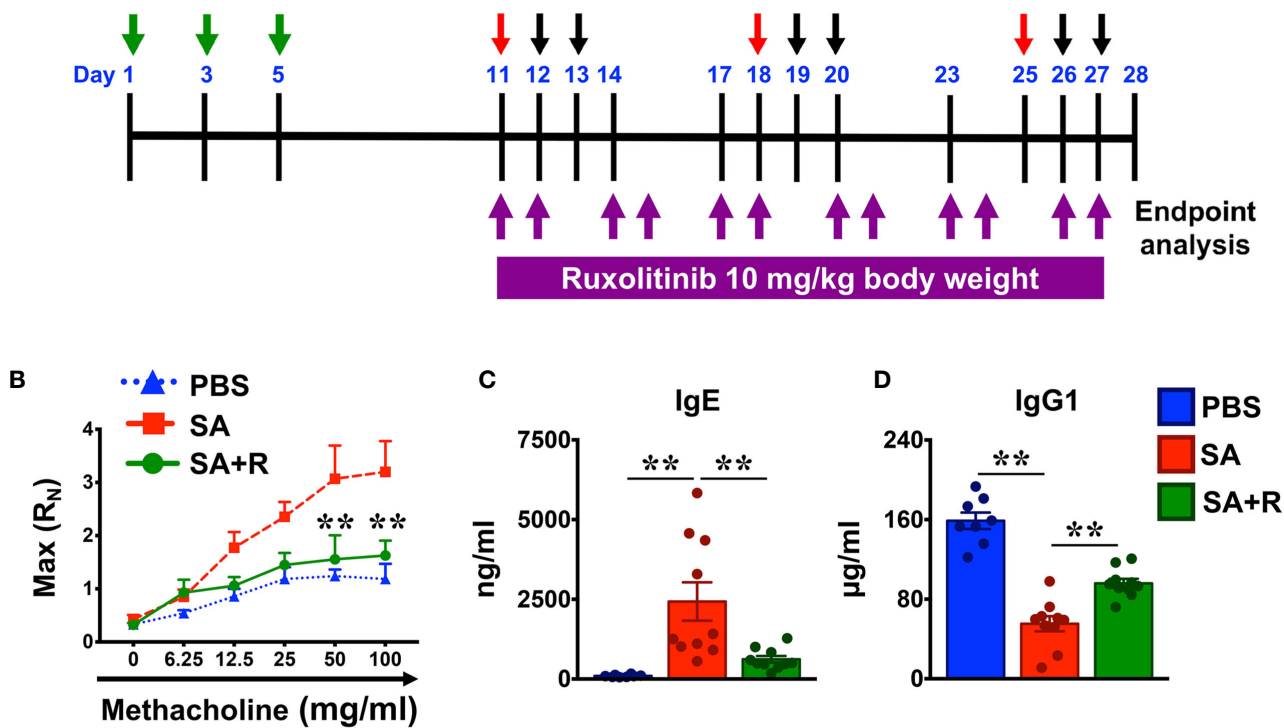

E

PBS

SA
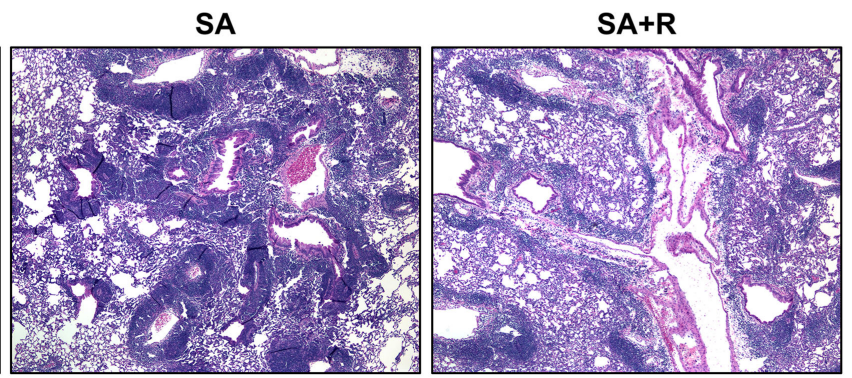

FIGURE 2 | Ruxolitinib ameliorates AHR as well as reduces IgE production and lung inflammation in a mouse model of SA. (A) Schematics of the severe asthma (SA) model used in the study. Mice were sensitized with HDME and c-di-GMP (green arrows) and subsequently challenged with HDME and a lower dose of c-diGMP (red arrows) or HDME alone (black arrors) as indicated. During the challenge phase, mice were also treated intranasally (i.n) with Ruxolitinib (R, $10 \mathrm{mg} / \mathrm{kg}$ body weight) for two consecutive days followed by a day of rest for a total of 12 injections. (B) Twenty-four hours after the last injection, mice were analyzed for AHR. Assessment of resistance to airflow in central airways (Newtonian resistance [Rn]) after challenge with increasing doses of methacholine (Mch). Data is shown as mean \pm SEM and pooled from 3 independent experiments with a total of 9-12 mice per cohort. Statistical significance was determined by two-way ANOVA. ${ }^{* *} \mathrm{p} \leq 0.01$. (C, D) Levels of serum IgE (C) and IgG1 (D) in PBS-treated, SA (challenged with HMDE+C-di-GMP) or SA mice treated with Ruxolitinib (SA+R). Data is shown as mean \pm SEM and pooled from 3 independent experiments with a total of 8-13 mice in each group. Statistical significance was determined by Student's unpaired $t$ test with Welch's correction. ${ }^{\star \star} \mathrm{p} \leq 0.01$. (E) Hematoxylin and eosin (H\&E) staining of lung section of PBS-treated, SA and SA+R mice. Scale bar: 100 $\mu$ m. Representative micrographs of the lung sections at $4 X$ magnification. Data is representative of 3 independent experiments with a total of 9 mice per cohort.

demonstrated significant elevation in $\mathrm{Rn}$, in a dose-dependent manner over a range of $6.25-100 \mathrm{mg} / \mathrm{ml}$ of Mch. In contrast, $\mathrm{SA}+\mathrm{R}$ mice manifested a dramatically decreased AHR, almost similar to levels observed in PBS-treated mice (Figure 2B).

Allergic asthma is characterized by elevated levels of serum $\operatorname{IgE}$ (10). Accordingly, we observed that serum IgE was significantly higher in SA mice as compared to the PBS controls (Figure 2C). IgE synthesis can occur through different biosynthetic pathways, either by "direct" class-switch recombination (CSR) from IgM, or through "sequential" switch from IgM to IgG1 and then from IgG1 to IgE (11). We observed that serum IgG1 levels were greatly reduced in SA mice
(Figure 2D), suggesting that HG induces class-switch of IgG1 to IgE. Importantly, Ruxolitinib treatment significantly decreased serum IgE but increased IgG1 levels in SA+R mice (Figures 2C, D). Thus it is likely that Ruxolitinib regulates IgE levels via direct and/or sequential CSR events.

Acute exposure to allergen increases AHR that is often associated with airway inflammation characterized by an influx of inflammatory cells (10). Having observed that Ruxolitinib attenuates HG-induced AHR, we next determined the effect of Ruxolitinib on lung inflammation. Histological analysis of the lung sections revealed that while $\mathrm{HG}$ induced airway inflammation in SA mice, it was substantially attenuated 
in Ruxolitinib-treated animals. This reduction in lung inflammation was most evident around the peribronchial and perivascular areas of the lung tissue sections (Figure 2E).

\section{Decreased Lymphocytic Infiltration in the Lungs of Ruxolitinib-Treated SA Mice}

To determine whether the decreased lung pathology observed in the $\mathrm{SA}+\mathrm{R}$ mice was due to a reduction in the recruitment of inflammatory cells to the airways, we harvested BALF and lung tissue and analyzed for total cell counts. We observed that following i.n. challenge with $\mathrm{HG}$, cellular infiltration in both the BALF and the lungs was significantly elevated. However, this was markedly reduced by Ruxolitinib (Figures 3A, B), which was consistent with the lung histology. To determine the relative makeup of the cellular inflammation, we used mutli-color flow cytometry and analyzed lung immune cells from PBS controls, $\mathrm{SA}$ and $\mathrm{SA}+\mathrm{R}$ mice (Figures $\mathbf{3 C}-\mathbf{K}, \mathbf{4}$ ). Increased $\mathrm{B}$ cell numbers are found in allergic lungs, as well as in the sputum of asthma patients $(12,13)$. Futhermore, while T lymphocytes, specifically $\mathrm{CD} 4+\mathrm{T}$-cells play a major role in the pathophysiology of asthma, particularly eosinophilic and CS-responsive asthma, CD8+ T cells are important effector cells in the pathogenesis of severe CSresistant asthma (14). Consistent with these reports, we observed that the cell numbers of all these lymphocytic populations were dramatically increased in the lungs of SA mice (Figures 3C-E). Invariant natural killer $\mathrm{T}$ (iNKT) cells are glycolipid-reactive innate $\mathrm{T}$ lymphocytes that play a critical role in the development of AHR (15). Accordingly, we observed that both the frequency and cell counts of iNKTs were increased in the lungs of SA mice (Figures 3F-H). In contrast, natural killer (NK) cell incidence was substantially reduced in these animals (Figures 3I, J). This was surprising as NK cells promote T cell-mediated acute allergic inflammation (16). Subsequently, when we evaluated these lymphocytic populations in $\mathrm{SA}+\mathrm{R}$ mice, we observed that Ruxolitinib had the most dramatic impact on the frequency and number of CD8+ T cells (Figures 3C-E), with concomitant decrease in B and NK cell counts (Figures 3E, K).

\section{Treatment With Ruxolitinib Reduced Macrophages and Eosinophils but Not Neutrophils in the Lungs of SA Mice}

Besides lymphocytes, several myeloid (dendritic cells) and granulocytes such as macrophages, eosinophils, and neutrophils are critical effectors of immune response in asthma (10). Macrophages are the most abundant immune cells in the lung and critically regulate environmental allergen-induced airway inflammation $(17,18)$. Phenotypically, macrophages can be $\mathrm{CD}_{11 \mathrm{c}^{+}}$SiglecF $^{+} \mathrm{MHCII}^{\text {high }}$ (M1) or $\mathrm{CD} 11 \mathrm{c}^{+}$SiglecF $^{+}$ $\mathrm{MHCII}^{\text {low }}$ (M2) sub-types that display inflammatory and antiinflammatory functions, respectively (18). We observed that while the frequency of $\mathrm{MHCII}^{\text {low }}$ (M2) macrophages were greatly reduced in SA mice, there was an increase in the both the incidence and total numbers of $\mathrm{MHCII}^{\text {high }}$ (M1) cells in these animals (Figures 4A-C). Consistent with our data, prior studies have linked M1 macrophages with the pathogenesis of SA, particularly for those with a poor response to systemic CS (19).
Next, we evaluated dendritic cells (DC) as they are principal antigen presenting cells and initiators of the immune response in allergic asthma (10). We observed a significant increase in the frequency and cell numbers of DC in SA mice (Figures 4A-C). Strikingly, while treatment with Ruxolitinib greatly reduced MHCII $^{\text {high }}$ (M1) cells in SA $+\mathrm{R}$ mice, it had little-to-no effect on the cell counts of MHCII ${ }^{\text {low }}$ (M2) macrophages or DC (Figure 4C). Finally, we examined the effect of Ruxolitinib on the frequency and cell counts of eosinophils and neutrophils (Figures 4D-I), key effector cells in T2-high and T2-low asthma endotypes, respectively (2). Interestingly, we observed that the incidence and absolute numbers of both these cells were significantly increased in SA mice, reflecting a mixed granulocytic inflammation. Importantly, while treatment with Ruxolitinib had no effect on either the frequency or cell numbers of neutrophils (Figures 4D-F), it significantly reduced eosinophilc infiltration in the lungs of $\mathrm{SA}+\mathrm{R}$ mice (Figures 4G-I).

\section{Ruxolitinib Targets Gene Expression of Several Chemokines but Not Alarmins in SA}

Alarmins such as thymic stromal lymphopoietin (TSLP), IL-25 and IL-33 can drive allergic inflammation in response to airway damage and their expression correlates with asthma severity (20). Surprisingly, we observed that gene expression of these alarmins in SA mice were either diminished (Tslp) or similar $(I l 25, I l 33)$ to PBS controls. Moreover, their expression was unaltered by Ruxolitinib treatment (Figure 5A). Several chemokines, particularly CCL5, CCL11, and CCL24 are known to regulate eosinophil trafficking to the lungs $(21,22)$. Consistently, we observed that the mRNA levels of Ccl5 and Ccll1 were significantly upregulated in SA mice; however, gene expression of $\mathrm{Ccl} 24$ was reduced in these animals (Figure 5B). Next, we examined chemokines that are known to promote the migration of neutrophils (CXCL1, CXCL2 and CXCL5) (22) and Th1 cells (CXCL10) (23) to the site of inflammation. We observed that gene transcripts of these chemokines were significantly upregulated in the lungs of SA mice (Figures 5C, D). However, while fold change (relative to PBS controls) in the gene expression of Cxcl1 and Cxcl2 ranged from $3.4 \pm$; 0.3 and $3.2 \pm ; 0.6$ respectively; mRNA levels of Cxcl5 (21.4 \pm ; 3.6) and Cxcl10 (32.6 \pm ; 4.1) were substantially higher. Of note, with the exception of Cxcl5, Ruxolitinib significantly suppressed the expression of all the other chemokines that were elevated in SA mice (Figures 5C, D).

\section{Ruxolitinib Suppresses Th1 and T2 but Not Th17 Cytokines in SA Mice}

CS-resistant severe asthmatics typically exhibit a T2-low asthma, wherein several pro-inflammatory cytokines including IFN- $\gamma$, TNF- $\alpha$, IL- 6 and IL-12 mediate its pathogenesis (1). So, we first assessed the gene expression of these cytokines and observed that with the exception of Il6, all the other pro-inflammatory cytokines were greatly increased in the lungs of SA mice (Figure 6A). Interestingly, gene expression of several T2 


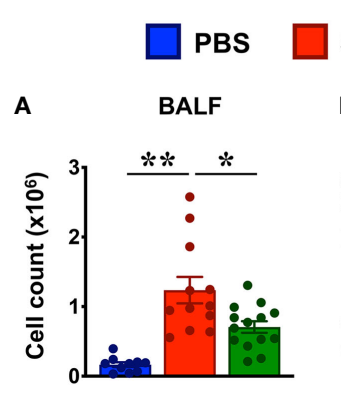

SA $\square$ SA+R
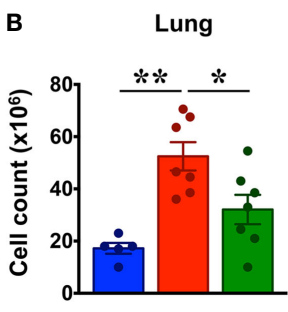

D
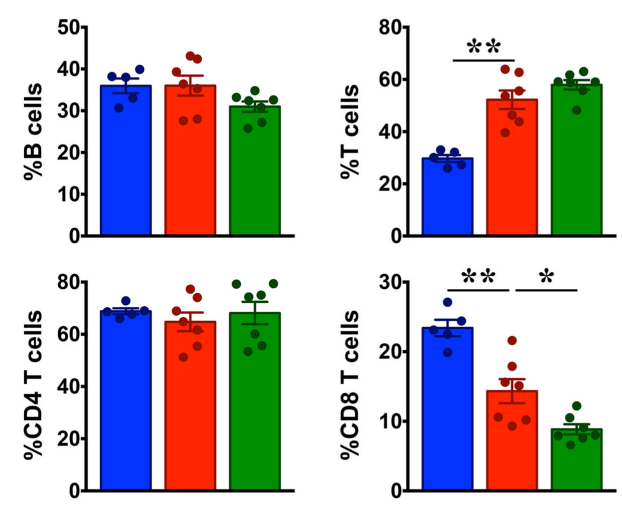

F

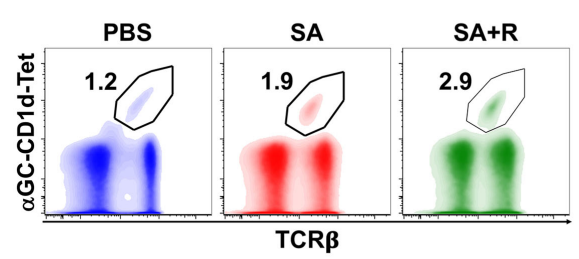

I

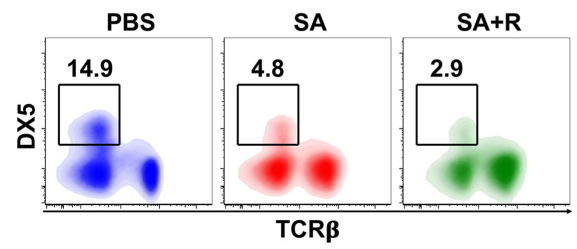

C

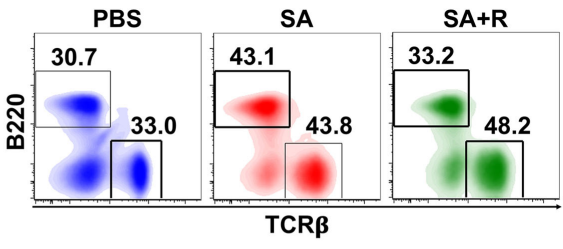

Gated on TCR $\beta+$ cells

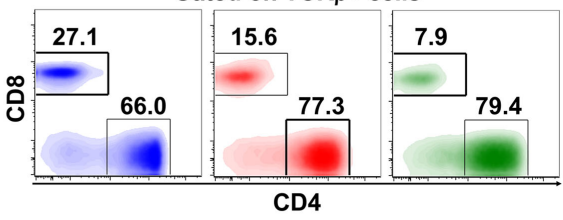

E
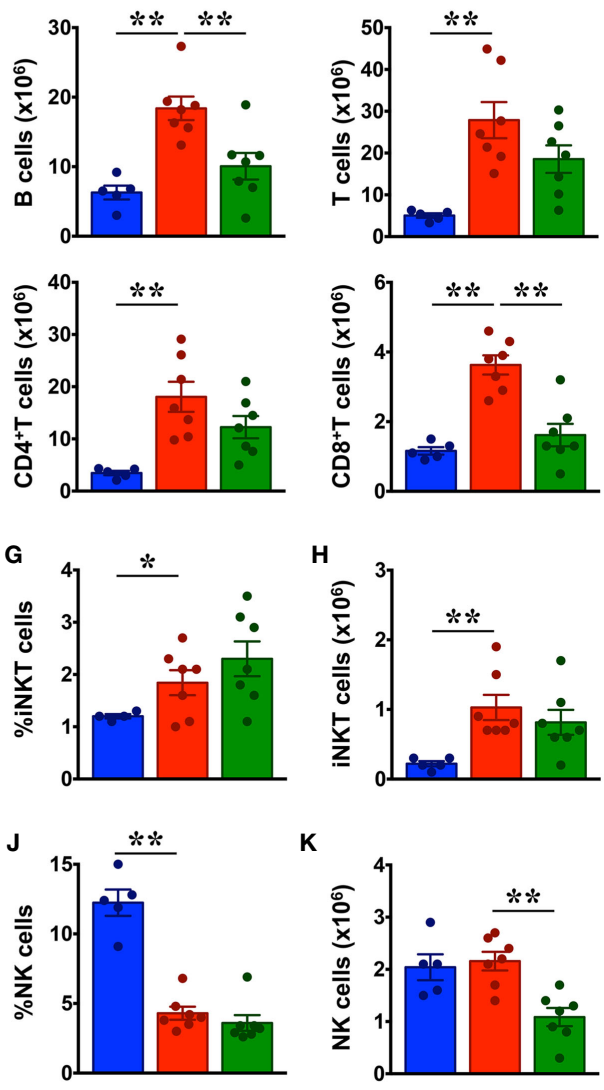

K

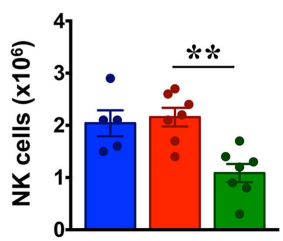

FIGURE 3 | Reduced cellular infiltration in the BALF and lungs of SA mice treated with Ruxolitinib. (A, B) BALF (A) and lung tissues (B) from control (PBS-injected), SA and SA mice treated with Ruxolitinib $(S A+R)$ were analyzed for total leukocyte counts. Data is shown as mean \pm SEM and pooled from 3 independent experiments with a total of 5-13 (BALF) or 5-7 (lungs) mice per cohort. (C-K) Lung immune cells were analyzed for various lymphocytic populations by flow cytometry. Representative density plots $(\mathbf{C}, \mathbf{F}, \mathbf{I})$ are from 2 independent experiments. Average frequency $(\mathbf{D}, \mathbf{G}, \mathbf{J})$ and total cell numbers $(\mathbf{E}, \mathbf{H}, \mathbf{K})$ of various lymphocytes from PBS, SA and SA+R mice were pooled. Data is presented as the mean of each group \pm SEM of at least 5-7 mice per group. Statistical significance was determined using Student's unpaired $t$ test with Welch's correction. ${ }^{*} p<0.05,{ }^{* *} p<0.01$.

cytokines such as Il4, Il5, Ill3 and Illo were also upregulated in these animals (Figure 6B). Importantly, while gene expression of most of these cytokines were significantly reduced by Ruxolitinib, mRNA levels of $I l 5$ and Il6 were comparable in SA and $\mathrm{SA}+\mathrm{R}$ mice (Figures 6A, B). IL-17A and IL-17F are members of the IL-17 cytokine family and are produced by
Th17 cells (24). In asthma patients, elevated levels of IL-17A and IL-17F are found in the airways and BALF that positively correlate with disease severity and neutrophil inflammation (1). Furthermore, IL-23 signaling is crucial for the maintenance and effector functions of Th17 cells (25). Consistent with these reports and increased neutrophilic 
A

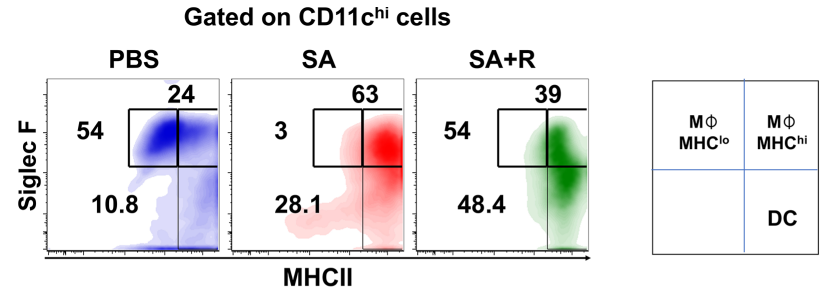

B
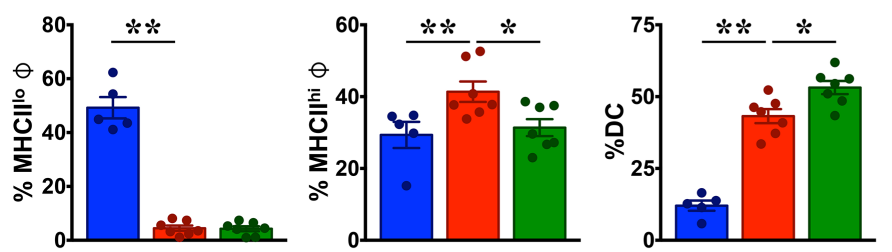

PBS

C
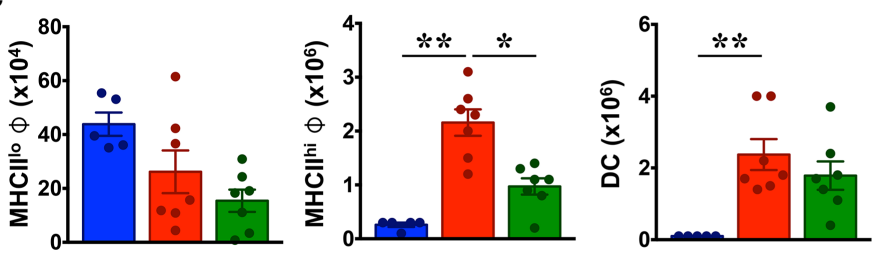

D
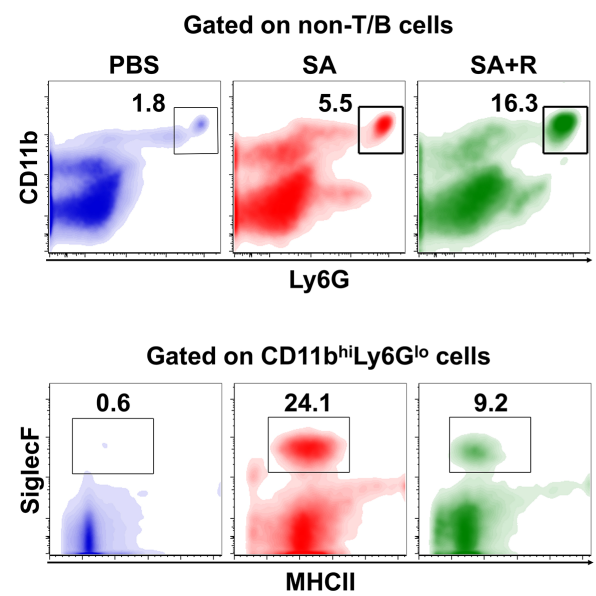

E
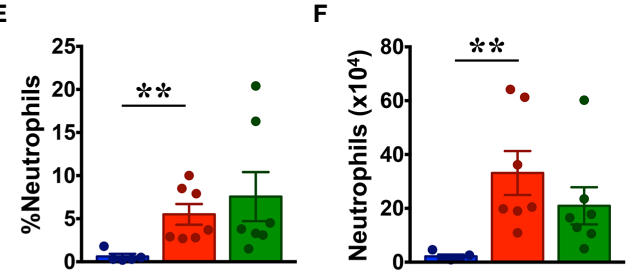

H

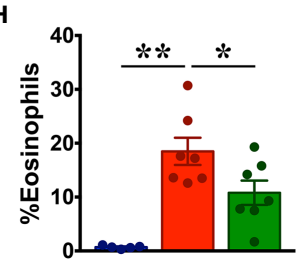

FIGURE 4 | Ruxolitinib reduces M1 macrophages and eosinophils but not neutrophils in the lungs of SA mice. (A-I) Lung cells were analyzed for various innate immune cell populations by flow cytometry. Representative density plots $\mathbf{( A ,} \mathbf{D}, \mathbf{G})$, average frequency (B, E, $\mathbf{H})$ and total cell numbers (C, $\mathbf{F}, \mathbf{I})$ of macrophages, dendritic cells (DC), neutrophils and eosinophils from PBS, SA and SA+R mice are shown. Data is pooled from 2 independent experiments and presented as mean \pm ; SEM of at least 5-7 mice per group. Statistical significance was determined using Student's unpaired $t$ test with Welch's correction. ${ }^{\star} p<0.05,{ }^{\star \star} p<0.01$.

infiltration (Figures 4D-F), we observed significantly elevated mRNA transcripts of $I l 17 a$ and $I l 17 f$ (Figure 6C) as well as $I l 23$ and Il23r (Figure 6D) in SA mice. However, while Ruxolitinib suppressed gene expression of Ill7f and Il-23r, Il17a and Il23 levels were comparable in SA and SA+R mice (Figures 6C, D).

To determine the effect of Ruxolitinib on the protein levels of Th1/Th2/Th17 cytokines, we cultured lung immune cells from $\mathrm{SA}$ and $\mathrm{SA}+\mathrm{R}$ in the presence of HG without or with ruxolitinb $(\mathrm{HG}+\mathrm{R})$ in vitro. We observed that lung immune cells from both $\mathrm{SA}$ and $\mathrm{SA}+\mathrm{R}$ mice produced robust levels of IFN- $\boldsymbol{\gamma}$ in the presence of HG that were significantly reduced in the presence of Ruxolitinib (Figure 7A). In contrast, SA mice produced modest amounts of IL-4 and IL-13 in the presence of HG and that were further suppressed in $\mathrm{SA}+\mathrm{R}$ mice (Figures $7 \mathbf{B}, \mathbf{C}$ ). Furthermore, both these cytokines were significantly reduced when cells were stimulated ex vivo in the presence of Ruxolitinib (HG+R, Figures 7B, C). Strikingly, while HG-stimulated cells from $\mathrm{SA}$ and $\mathrm{SA}+\mathrm{R}$ mice produced comparable IL-17A, these levels were significantly increased when cells were cultured in presence of $\mathrm{HG}+\mathrm{R}$ (Figure 7D). 
A

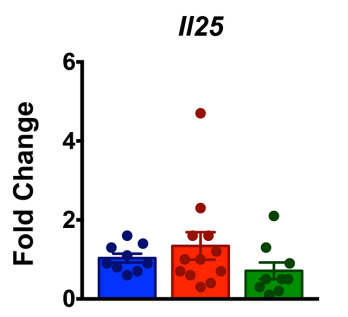

B

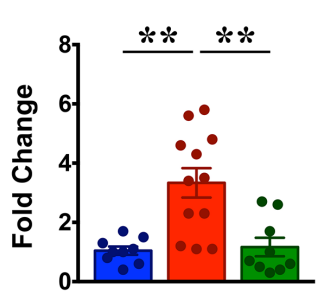

C

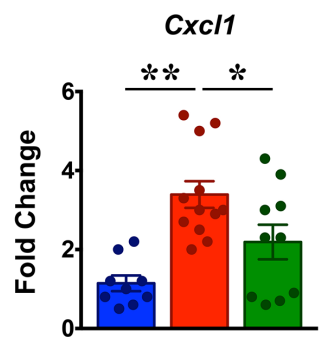

$1 / 33$

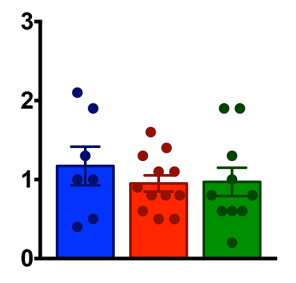

Cc/11

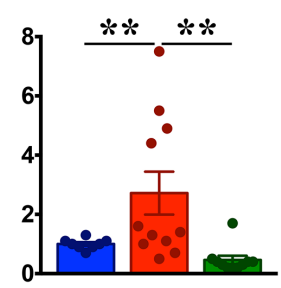

Cxc/2

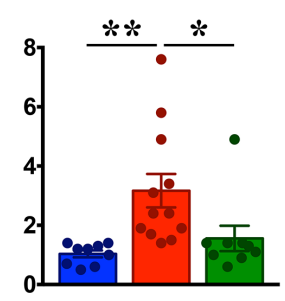

Tslp

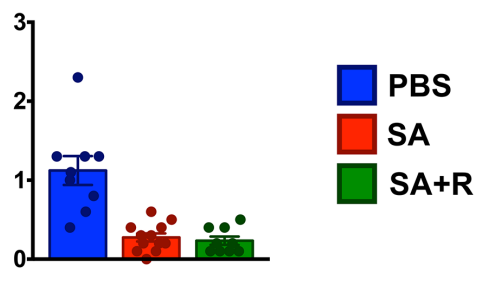

Ccl24

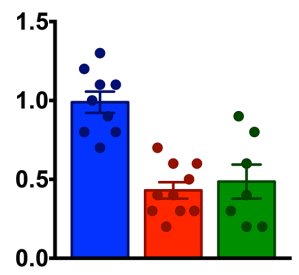

FIGURE 5 | Reduced chemokine expression in the lungs of Ruxolitinib-treated SA mice. (A-D) Lungs tissues of PBS-treated, SA and SA+R mice were analyzed for gene expression of (A) alarmins (//25, //33, Ts/p), and various eosinophilic (B), neutrophilic (C), and Th1 (D) chemokines by qPCR. Data is shown as mean fold change \pm SEM and pooled from 3 independent experiments with a total of 8-12 mice per cohort. Statistical significance was determined by Student's unpaired $t$ test with Welch's correction. ${ }^{\star} p \leq 0.05,{ }^{* *} p \leq 0.01$.

\section{Ruxolitinib-Treated SA Mice Exhibit Reduced Expression of NLRP3, IL-1 $\beta$ and Mucins}

Excessive nucleotide-binding oligomerization domain-like receptor family, pyrin domain-containing 3 (NLRP3) inflammasome and concomitant IL- $1 \beta$ responses play a critical role in steroid-resistant SA (26) and promote Th17 allergic asthma in mice (27). Given that SA+R mice had elevated levels of IL-17A, we determined the effect of ruxoltinib on the gene expression of NLRP3 and IL-1 $\beta$. Consistent with the welldocumented role for the NLRP3-IL-1 $\beta$ axis in the pathogensis of SA (28), we observed increased gene transcripts of these molecules in the lungs of mice challenged with $\mathrm{HG}$ (Figure 7E). In conjunction with IL-1 $\beta$, TGF- $\beta$ also promotes Th17 development (25). Accordingly, gene expression of Tgf $\beta$ was also increased in SA. Importantly, mRNA expression Nlrp3, $I l 1 \beta$ and $T g f \beta$ were significantly reduced in $\mathrm{SA}+\mathrm{R}$ mice (Figures 7E, F), suggesting that the persistent high IL-17A levels in these mice was independent of the NLRP3-IL-1 $\beta$ axis as well as TGF- $\beta$. Furthermore, gene expression of Roryt (lineage-defining transcription factor of Th17 cells) (25) was neither elevated in SA nor altered in SA+ R mice, as compared to levels in PBS controls (Figure 7F).

T-bet is a critical regulator of Th1 lineage commitment and IFN- $\gamma$ production (29). In contrast, secretory leukocyte protease inhibitor (SLPI) negatively regulates IFN- $\gamma 3$. However, we observed that the mRNA levels of both Tbet and Slpi were increased in SA mice relative to PBS controls. Interestingly, while mRNA expression of Tbet was suppressed, Slpi gene transcript was significantly increased in $S A+R$ mice (Figure 7G).

The direct downstream target of IL-13 and to some extent of IL-4, is mucus production by goblet cells in the airways (30). Goblet cell hyperplasia partly contributes to airway remodeling by mucus hypersecretion and airflow obstruction (31). In mice, excess mucus production is regulated by 2 polymeric mucins: Muc5ac and Muc5b (31). We observed that both Muc5ac and Muc5b gene transcripts were significantly upregulated in the lungs of SA mice. Importantly, in agreement with reduced IL-4 and IL-13 levels in SA $+\mathrm{R}$ mice, mRNA levels of both $M u c 5 a c$ and $M u c 5 b$ were markedly reduced by treatment with Ruxolitinib (Figure $\mathbf{7 H}$ ). 


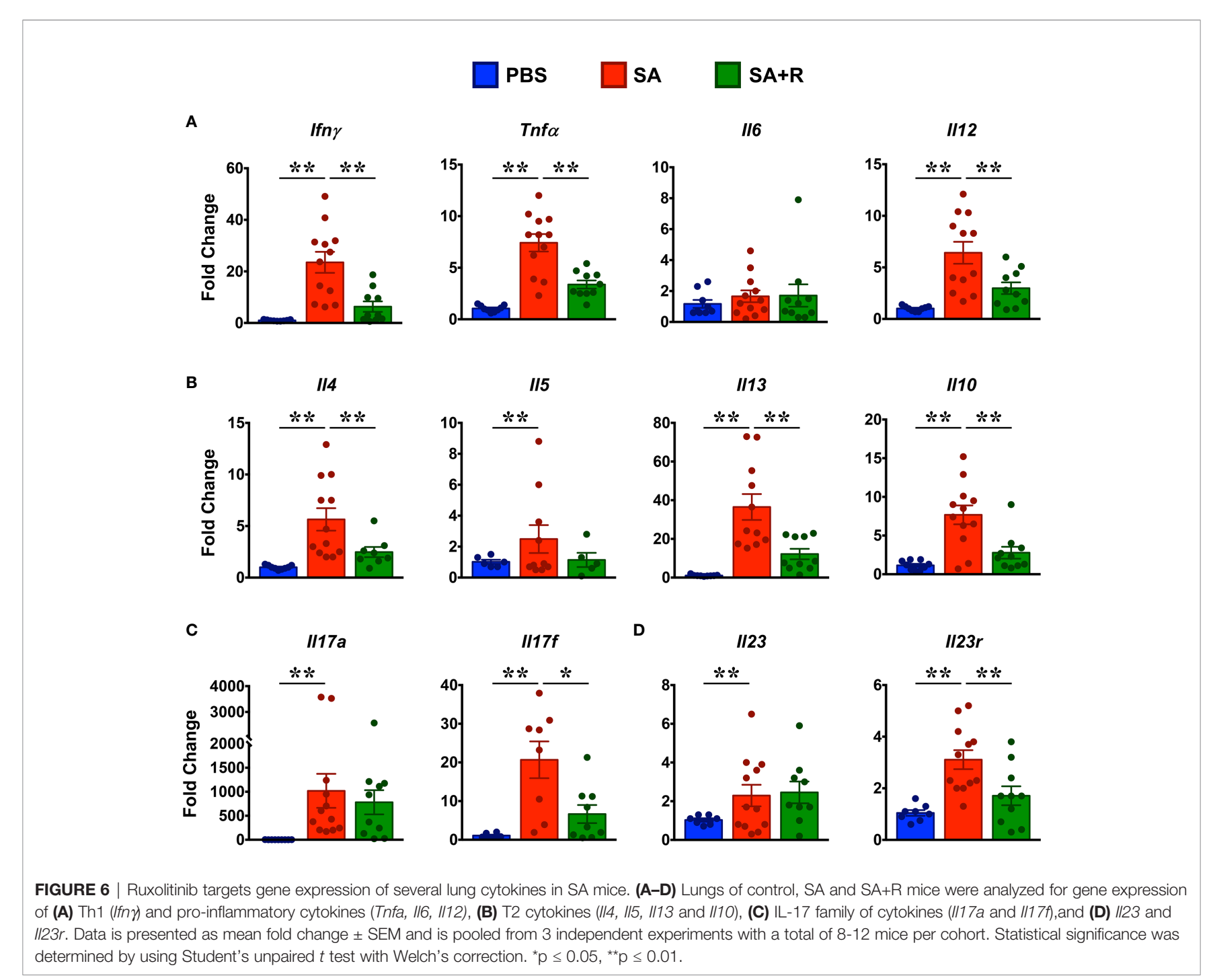

\section{Ruxolitinib Regulates miRNA Expression in the Lungs of SA Mice}

Several microRNAs (miRNAs) play either a protective or proinflammatory role in asthma (32). As depicted in the heat map, we screened for various miRNAs that have known roles in different aspects of asthma pathogenesis (Figure 8A). We observed that while PBS controls and SA mice had comparable levels of several miRNAs including miR23b-3p, miR126-5p, miR221-5p and, miR223-3p (Figure 8B), expression of miR215p, miR142-3p, miR150-5p, miR155-3p and miR155-5p were significantly increased in SA mice (Figures $\mathbf{8 C}$ ). In contrast, gene transcripts of miR126-3p, miR145-5p, miR206-5p, miR495-5p and miR708-5p were greatly reduced in SA mice as compared to the PBS controls (Figures 8D). Importantly, expression of these miRNAs were significantly increased in SA+R mice (Figures 8BD). Additionally, we observed an upward trend in the expression of most Let-7 miRNAs in these mice (Figure 8A). As several of these miRNAs have established roles in airway smooth muscle cell (ASMC) proliferation, AHR and lung inflammation, our data suggest that treatment with Ruxolitinib suppresses the pathogenesis of SA, at least in part via regulation of miRNAs.

\section{DISCUSSION}

Traditional therapy for asthma includes inhaled or systemic CS, bronchodilators, and anti-leukotrienes $(1,33)$. However, asthma is a complex heterogeneous disease with varying phenotypes and endotypes $(1,2,33)$. As such, traditional therapies do not cater to all the asthma patients. Furthermore, prolonged use of CS may lead to several adverse effects including heart disease, infections, osteoporosis, bone fracture, stroke, and cataract (2). Importantly, $5-10 \%$ patients are refractory to mainstay asthma treatments and contribute significantly to the morbidity and mortality associated with asthma (33). These subjects have SA and exhibit a high Th1/ IFN- $\gamma$ immune response in spite of ongoing CS treatment (1). The emergence of new biologics offers alternative options to improve asthma control. While some of these targeted therapies 


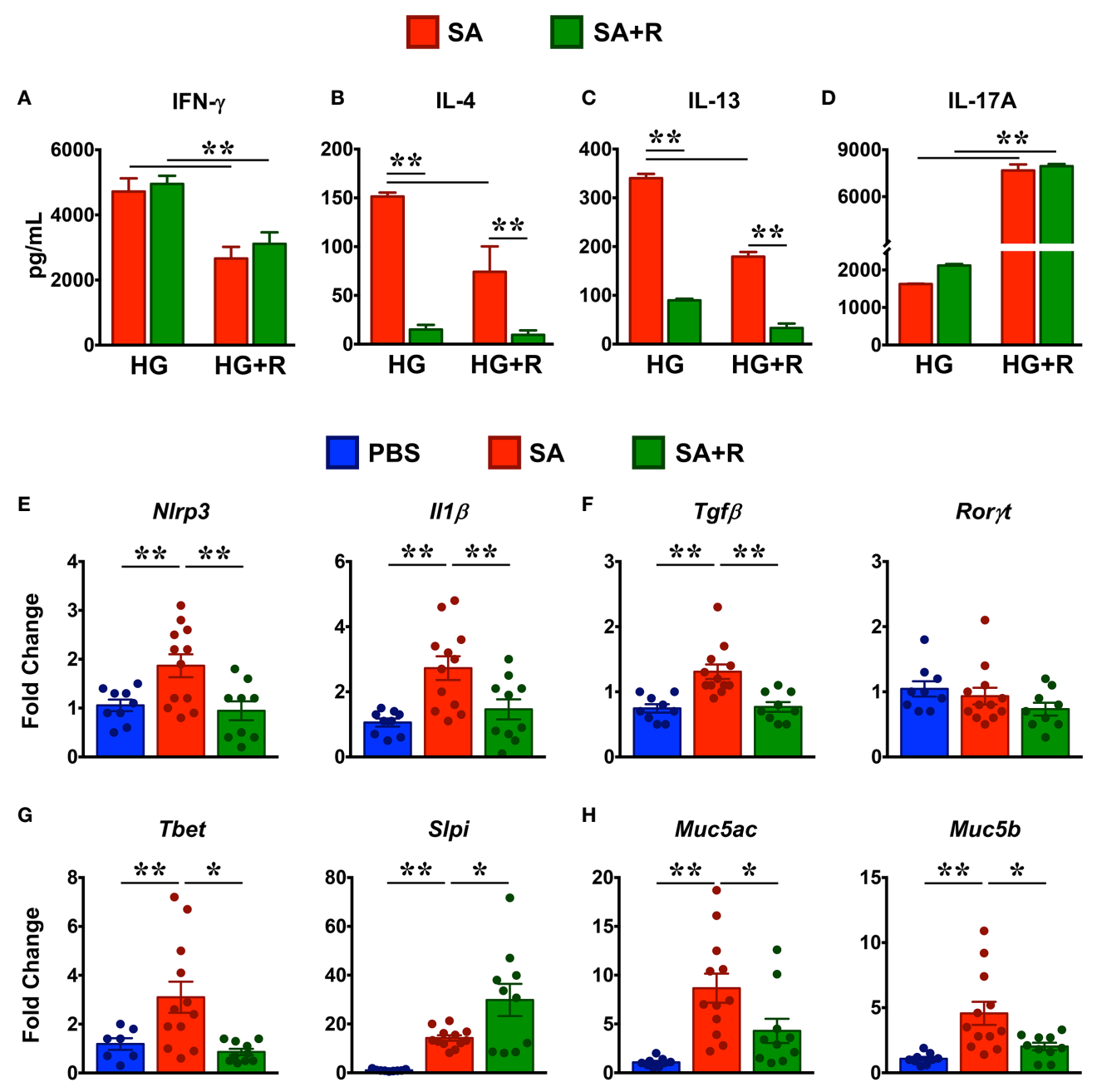

FIGURE 7 | Treatment with Ruxolitinib suppresses Th1/Th2 cytokine production and associated gene expression in SA mice. (A-D) Lung immune cells from SA and SA+ $\mathrm{R}$ mice were isolated and cultured ex vivo in the presence of either $\mathrm{HMDE}+\mathrm{C}-\mathrm{di}-\mathrm{GMP}(\mathrm{HG})$ or $\mathrm{HMDE}+\mathrm{C}-\mathrm{di}-\mathrm{GMP}+\mathrm{Ruxolitinib}(\mathrm{HG}+\mathrm{R})$, as indicated. After 72 hours, cell culture supernatants were analyzed for IFN- $\gamma(\mathbf{A}), I L-4$ (B), IL-13 (C), and IL-17A (D). Data is shown as mean $\pm S D$ and is from 1 representative experiment with $n=3$ mice per cohort. Statistical significance was determined by Student's unpaired $t$ test with Welch's correction. ** $\mathrm{p} \leq 0.01$. (E-H) Lung tissues from PBS-treated, SA and SA+R mice were analyzed for gene expression of (E) inflammasome pathway (N/rp3 and II1 $\beta$ ), (F) IL-17-associated genes (Tgf $\beta$ and Ror $\gamma$ ) (G) IFN- $\gamma$-regulating genes (Tbet and S/pi), and $\mathbf{( H )}$ polymeric mucins (Muc5ac and Muc5b). Data in (E-H) are presented as mean fold change \pm SEM and are pooled from 3 independent experiments with a total of 9-12 mice per cohort. Statistical significance was determined using Student's unpaired $t$ test with Welch's correction. ${ }^{\star} p \leq 0.05$ or ${ }^{* \star} p \leq 0.01$.

seem promising, others have potential limitations $(2,20)$. For example, FDA-approved anti-IgE monoclonal antibody (mAb), is applicable for a subset of patients that have uncontrolled IgEmediated allergic asthma. Other biologics include mAbs against IL-5, IL-4 and IL-13 and their respective cognate receptors. However, these mAbs primarily target immune cells and cytokines implicated in the T2-high inflammation. Several cytokines are pivotal in the regulation of airway inflammation and tissue remodeling by their actions on immune, epithelial and ASMCs (33). Thus, targeting a single cytokine or inflammatory cell may not be sufficient to reduce inflammation, possibly because of compensatory response from a different cytokine. Therefore, developing drugs that can suppress multiple cytokines may be a more effective strategy.
As most pro-inflammatory cytokines signal through the JAKSTAT pathway (4), JAK inhibition is a potential therapeutic target for the treatment of asthma $(5,6)$. Indeed, prior studies have shown that several oral JAK inhibitors can reduce airway and lung inflammation in ovalbumin (OVA)-induced murine model of asthma (34-36). However, prolonged use of oral JAK inhibitors increase the risk of systemic side effects such as lymphopenia, neutropenia, infections, intestinal damage, liver dysfunction as well as malignancies (37). Moreover, the effect of JAK inhibition on CS-resistant SA remained to be evaluated. To directly address these issues, in the current study, we determined the effect of inhaled JAK inhibition on the immunopathology of CS-resistant SA.

Our studies reveal several important observations. First, Ruxolitinib reduces AHR by downregulating IFN- $\gamma$ but not IL- 
A

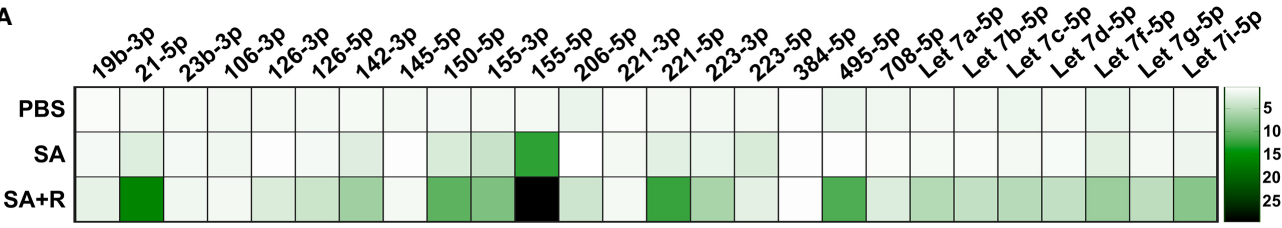

B
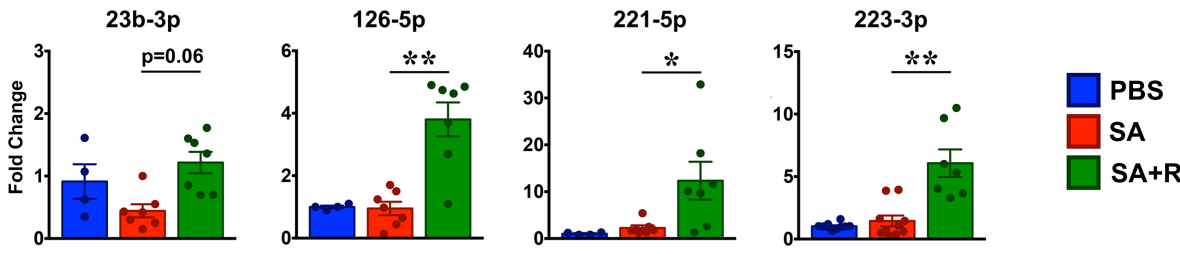

C
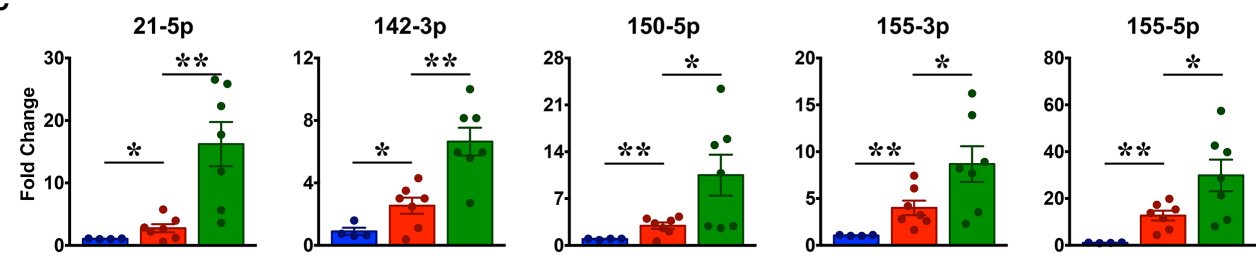

D
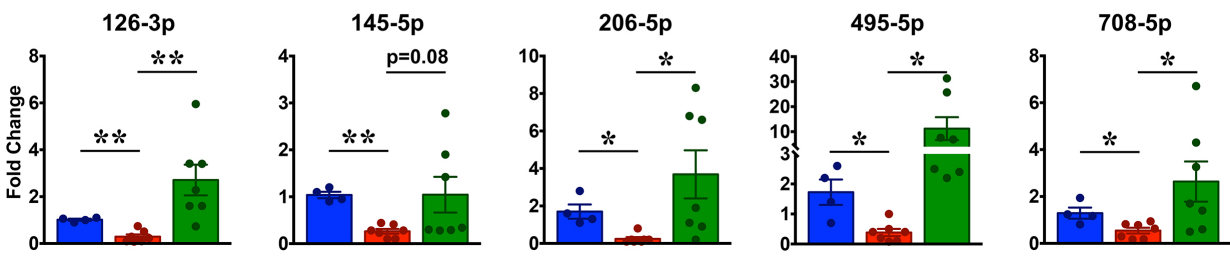

FIGURE 8 | Increased expression of various miRNAs in the lungs of SA mice treated with Ruxolitinib. Lungs tissues of PBS-injected, SA and SA+R mice were analyzed for gene expression of various miRNAs as indicated in the figure. (A) Heat map of gene expression of various miRNAs in the lungs of SA and SA+R mice, normalized to PBS controls. (B-D) Fold change in expression of various miRNAs in the lungs of SA and SA+R mice with known roles in asthma pathogenesis. Fold change for each cohort was normalized to PBS-treated mice. Data is presented as mean \pm SEM and are pooled from 2 independent experiments with a total of $n=4$ mice for PBS controls and $\mathrm{n}=7$ mice for SA and SA+R groups. Statistical significance was determined using Student's unpaired $t$ test with Welch's correction. * $\mathrm{p} \leq$ 0.05 or ${ }^{\star \star} \mathrm{p} \leq 0.01$.

17A cytokine levels. Indeed, previously it has been shown that IFN- $\gamma$ but not IL-17A promote AHR (3). In the same study, the authors demonstrate an inverse correlation between IFN- $\gamma$ and SLPI and that increased SLPI levels attenuate AHR (3). However, it is worth noting that SA patients exhibit elevated levels of SLPI, albeit at significantly lower extent than those observed in mildmoderate asthmatics (3). Consistently, we observed that although Slpi is significantly increased in SA, its expression is considerably lower when compared to $\mathrm{SA}+\mathrm{R}$ mice. Interestingly, SLPI negatively regulates the activation of TGF- $\beta$ (38), a key mediator of airway remodeling during asthma progression (39). It is well established that TGF- $\beta$ promotes aberrant proliferation and proinflammatory effects of ASMC, the principal effectors of AHR $(40,41)$. Additionally, TNF- $\alpha$ and IL-13 can also promote AHR by either directly acting on ASMC or by inducing the release of various inflammatory mediators (42) or by augmenting mucus secretion that results in thickening of the airways (30). As the expression of $T g f \beta$, Tnf $\alpha$ and $I l 13$ as well as its downstream targets, Muc5ac and Muc5b were dampened by Ruxolitinib, our data indicate that JAK1/2 inhibition ameliorates AHR by affecting multiple pathways.

Second, Ruxolitinib alters the lung immune landscape and inhibits the expression of several mediators that can amplify and/ or perpetuate the Th1 inflammation associated with CS-resistant SA. To that end, CD8+T, iNKTs, DCs and M1 macrophages were elevated in SA mice. This was accompanied by increased expression of Tbet, several proinflammatory cytokines (Ifng, Il12, Tnfa, and Il1 $)$, and the chemokine Cxcl10. T-bet is not only a potent inducer of IFN- $\gamma$ (43) but is also critical for Th1/ Th2 differentiation of CD4+ T cells $(43,44)$. It is also expressed by antigen-presenting cells (APCs), including DCs for optimal production of IFN- $\gamma$ and subsequent antigen-specific Th1 cell activation $(45,46)$. It is interesting to note that while T-bet was increased in SA mice, CD8+ $\mathrm{T}$ cells were not as numerous as $\mathrm{CD} 4+\mathrm{T}$ cells in the lungs of these animals. However, as both CD4+ and CD8+ $\mathrm{T}$ cells were increased in SA mice, our data highlight a co-operative role for these $\mathrm{T}$ cell subsets in AHR and airway inflammation. Indeed, prior studies have shown that 
CD4+ $\mathrm{T}$ cells are required for CD8 $+\mathrm{T}$ cell activation (47) and when co-transferred at the same time, they drive the most severe airway response (48).

IL-12 is produced by activated monocytes, macrophages, and DCs that direct the differentiation of T cells into Th1 cytokineproducing cells while suppressing Th2 polarization (10). Additionally, IFN- $\gamma$ and IL-12 promote M1 macrophage differentiation that in turn produce more Th1 cytokines including TNF- $\alpha$, IL-1 $\beta$, IL-6, and IL-12, establishing a positive feedback loop $(17,18)$. Furthermore, IL-12 augments NK and $\mathrm{CD} 8+\mathrm{T}$ cell proliferation as well as IFN $-\gamma$ production by these cells (49). TNF- $\alpha$ is a pleiotropic cytokine that recruits various inflammatory cells into the lung tissue and activates them to produce several proinflammatory cytokines (including TNF- $\alpha$ itself) and chemokines (50). Notably, TNF- $\alpha$ and IFN- $\gamma$ individually or synergistically can enhance CXCL10 production, a strong chemoattractant for Th1 cells (50). Consistently, CXCL10 is elevated in the BAL cells of CS-resistant SA patients (23), as observed in our experimental mice. Except for IL-6, Ruxolitinib significantly reduced the expression of all these inflammatory mediators. Accordingly, the immune populations (CD8+ T, NK and M1 macrophage) producing these cytokines and/or being affected by them, were dramatically fewer in the lungs of SA+R mice. In contrast, DC numbers were not affected by Ruxolitinib. As DC are the major source of IL-12 and TNF- $\alpha 10$, our studies indicate that JAK1/2 inhibition primarily impairs DC functions. Indeed, a prior study has shown that Ruxolitinib affects DC functions leading to impaired $\mathrm{T}$ cell activation (51).

Third, Ruxolitinib suppresses the T2 immune response associated with CS-resistant SA. This is evident by reduced lung eosinophilia and IgE production in SA+R mice. Elevated IgE levels in the serum is a hallmark feature of allergic asthma (10), where T2-cytokines IL-4 and IL-13 promote B cell differentiation, isotype class switching and IgE secretion (10). Moreover, in the presence of IL-4, IL-10 a major regulatory cytokine enhances B cell proliferation and IgE synthesis (52). Thus, the reduced levels of serum $\operatorname{IgE}$ in $\mathrm{SA}+\mathrm{R}$ mice can be attributed to the diminished expression of these T2 cytokines as well as to the significantly fewer B cell numbers in lungs of these mice. Airway eosinophilia is not only characteristic of T2-high asthma but is also present in T2low asthma (1). Indeed, steroid-insensitive patients with eosinophil-enriched inflammation have been described (53), consistent with our data. IL-5 is a key cytokine for the differentiation, activation and survival of eosinophils and together with IL-4 and IL-13, it regulates the recruitment of these cells into the lungs (54). As Il5 mRNA levels were unaffected by Ruxolitinib, yet eosinophilia was greatly reduced in SA+R mice, our observations suggest that both IL-4 and IL-13 drive eosinophilic inflammation in the lung. However, IL-13 has been touted as the key mediator of T2-dominant immune responses $(30,54,55)$, perhaps due to its greater production and persistence (56). Indeed, we observed that IL-13 was much higher than IL-4 in the lung tissue and culture supernatants from SA mice, akin to data from CS-resistant SA patients (3).

Several chemokines, particularly CCL5, CCL11, and CCL24 are also involved in the airway recruitment of eosinophils (22).
Strikingly, we observed that $\mathrm{Ccl} 5$ and $\mathrm{Ccl11}$ but not $\mathrm{Ccl} 24$ levels were increased in SA mice. CCL5 and CCL11 are both highly expressed by airway epithelial cells, however, CCL5 is produced by Th1 and CCL11 by Th2 cells (22). Importantly, CCL5 can induce receptor-mediated calcium flux and chemotaxis while CCL11 primarily mediates eosinophil degranulation (22). Additional studies have shown that CCL5 plays a role in the regulation of airway function through modulation of IFN- $\gamma$ and IL-12 levels (57) and that both these chemokines contribute to the irreversible airflow obstruction in SA (58). Given that both Ccl5 and Ccl11 were dramatically reduced in SA+R mice, our studies reveal that JAK1/2 inhibition can ameliorate lung inflammation not only by targeting eosinophil chemotaxis and function but also by interfering with the cross-regulation of the Th1 and T2 inflammation that co-exist in steroidinsensitive patients.

Fourth, Ruxolitinib impairs the NLRP3-IL-1 $\beta$ axis but fails to resolve the neutrophilic infiltration observed in SA mice. Activation of NLRP3 inflammasome leading to IL-1 $\beta$ secretion contributes to lung inflammation via Th17 differentiation and IL17 production $(26,28)$. Consistent with this notion, we observed increased expression of Nlrp3, Il1 $\beta$ as well as Il17a and Ill7f in SA mice. However, while Nlrp3, Ill $\beta$ and Il17f levels were significantly reduced in SA+R mice, Ill7a remained unaltered, suggesting that either NLRP3 does not regulate IL-17A production in our model or that there are additional mechanisms at play. Alternatively, it is also possible that NLPR3 contributes to the airway inflammation independent of IL-17A. Indeed, NLRP3-deficient mice exhibit dramatically reduced eosinophil recruitment, mucus secretion and IL-1 $\beta$ that correlate with reduced Th2 cytokine and chemokine production (59). Several cytokines including IL-6, TGF- $\beta$, IL- $1 \beta$ and IL-23 promote differentiation of CD4+T cells to Th17 cells (25). Another source of IL-17A in the lungs is iNKT cells (15). As both CD4+ T and iNKT cell numbers were preserved in $\mathrm{SA}+\mathrm{R}$ mice, these cells are likely to contribute to the sustained IL-17A levels in our model. Surprisingly, we observed very little Il6 in the lungs of SA mice, however, it is possible that IL- 6 is elevated during the sensitization phase and reduces to baseline levels in the challenge phase. Whether these or other mechanisms regulate the IL-6-IL-17 axis in the SA mice remain to be evaluated.

IL-17 plays a critical role in neutrophil recruitment to the lungs by regulating expression of several chemokines, including CXCL1, CXCL2 and CXCL5 (28); all of which were elevated in SA mice. Interestingly, expression of $\mathrm{Cxcl} 1$ and $\mathrm{Cxcl} 2$ was decreased by Ruxolitinib, yet neither the frequency nor the number of neutrophils were reduced in SA+R mice. Notably, like Il17a, CXcl5 was unaffected by Ruxolitinib and its expression was much higher than $\mathrm{Cxcl} 1$ and $\mathrm{Cxcl} 2$ indicating that lung neutrophilia observed in the SA+R mice is mediated by the IL-17A-CXCL5 axis. Functionally, neutrophils can be proinflammatory or immunosuppressive and can exert adverse or beneficial effects in asthma (60). Given the considerable attenuation of disease severity in our model, we favor a protective role for these cells. Indeed, a protective role for neutrophils in limiting HDME- induced allergic airway inflammation has been reported (61). Contrary to our observations, a recent study demonstrated that Ruxolitinib can 
reduce neutrophilic inflammation (62). This discrepancy is attributable to the differences in the allergens used as well as the route, dose and timing of Ruxolitinib administration. Indeed, we demonstrate that Ruxolitinib can significantly decrease or increase IL-17A levels in culture supernatants from lung cells, depending on whether the cells were stimulated after the sensitization (Figure 1D) or the challenge phase (Figure 7D), respectively.

Finally, we demonstrate that Ruxolitinib upregulates the expression of several miRNAs linked to multiple aspects of asthma pathogenesis. We observed that of the few miRNAs that were elevated in the lung tissues of SA mice, miR155-5p was most highly upregulated. Indeed, studies have shown that miR155 overexpression promotes Th1 responses upon IFN- $\gamma$ stimulation (63), induces a pro-inflammatory M1 macrophage response, and enhances IL-12 production by DC (64). Additionally, it can directly target the transcription factor PU.1. to promote Th2 allergic inflammation (65). Surprisingly, of the many miRNAs that were upregulated in the $\mathrm{SA}+\mathrm{R}$ mice, miR155-5p was among the most highly expressed, even beyond levels seen in SA mice. As Ruxolitinib significantly ameliorated both T1 and T2 immune response, it is reasonable to speculate that higher miR155-5p expression in $\mathrm{SA}+\mathrm{R}$ confers protection. Consistent with our hypothesis, studies have shown that low miR155 expression favors IL-1 $\beta$ production, however high miR155 levels suppress gene expression of IL-1 and other proinflammatory cytokines (66). Importantly, miR155 inhibits IFN- $\gamma$ signaling in CD4+ T cells (67) and also downregulates T2 responses by directly targeting the transcription factor, c-Maf (68) and IL-13 receptor $\alpha 1$ (69).

Increased expression of several miRNAs inhibit ASMC proliferation or AHR via different pathways. Specifically, miR23b-3p (70) and miR142-3p (71) inhibit TGF $\beta$-dependent ASMC proliferation while miR150-3p and miR708-5p inhibit ASMC proliferation by downregulating BCYRN1 (72) and CD38 (73), respectively. Moreover, miR145-5p alleviates airway remodeling by targeting EGFR to downregulate Muc5ac expression (74). On the other hand, several miRNAs promote T2 effector functions. For example, miR-21 potentiates Th2 polarization by suppressing Th1 differentiation $(75,76)$, miR126 promotes eosinophil recruitment (77) and miR206 upregulates airway IL-25 and TSLP levels (78). In contrast, Let-7 miRNAs, miR150 and miR495 downregulate T2 responses by targeting IL-13 (79) or the transcription factor, GATA-3 $(80,81)$. Additionally, while miR221 promotes Th1 inflammation (64), miR223 inhibits the NLRP3/IL-1 $\beta$ axis (82) as well as granulocyte progenitor cell differentiation and activation (83). Strikingly, all these miRNAs were increased in $\mathrm{SA}+\mathrm{R}$ mice. Collectively, our data underscore the complexity of the miRNA regulatory networks that co-operate with or cross regulate each other to ameliorate AHR and lung inflammation. However, the exact mechanism by which Ruxolitinib impacts expression of these miRNAs in various immune and nonimmune cells, warrants further investigation.

In conclusion, we demonstrate that intranasal administration of low dose Ruxolitinib dramatically reduces AHR, lung inflammation and IgE production in a mouse model of SA. This amelioration is linked to the suppression of several chemokines, cytokines, and downstream inflammatory mediators that drive asthma pathogenesis in steroid-unresponsive patients. Ruxolitinib is a potent, highly bioavailable JAK1/2 inhibitor that is already US Food and Drug Administration (FDA) approved for the treatment of myelofibrosis, polycythemia vera, steroid-refractory acute graft versus host diseases and atopic dermatitis (84). Importantly, Ruxolitinib targets inflammation differently than steroids, and thus, it can be used independently or in combination with steroids to treat severe asthmatics more effectively. Recently, two reports have shown that local inhibition of JAK in the lung suppresses OVA-induced lung inflammation and $\operatorname{AHR}(85,86)$. Together with our data, these studies provide evidence that JAK inhibition by the inhaled route provides a promising alternative for both mild and severe asthma treatment.

\section{DATA AVAILABILITY STATEMENT}

The original contributions presented in the study are included in the article/Supplementary Material. Further inquiries can be directed to the corresponding author.

\section{ETHICS STATEMENT}

The animal study was reviewed and approved by Institutional Animal Care and Use Committee at the Michigan State University.

\section{AUTHOR CONTRIBUTIONS}

TH performed experiments and analyzed data. AK performed experiments and assisted with AHR data analysis. DB performed FACS staining, ELISA and qPCR for miRNA analysis. KT performed ELISA and assisted in organ processing for $\mathrm{qPCR}$ and histology. HS and RD designed and supervised the research, analyzed the data and wrote the manuscript. All authors read and edited the article and approved the submitted version.

\section{FUNDING}

This work was supported by grants from the National Institutes of Health to RD (5K22CA18814802) and HS (5R00HL12107305). KT was a NIH-NHLBI scholar, her research training was supported through an NIH 5-R25-HL108864-award to Dr. Elahě Crockett, Director of the Research Education Program to Increase Diversity in Health Researchers (REPID), Michigan State University.

\section{ACKNOWLEDGMENTS}

The authors thank the National Institutes of Health (NIH) Tetramer Core Facility for providing the fluorochrome conjugated CD1d-tetramer (CD1d-Tet) loaded or unloaded with glycolipid antigen (PBS57). 


\section{REFERENCES}

1. Ray A, Raundhal M, Oriss TB, Ray P, Wenzel SE. Current Concepts of Severe Asthma. J Clin Invest (2016) 126:2394-403. doi: 10.1172/JCI84144

2. Russell RJ, Brightling C. Pathogenesis of Asthma: Implications for Precision Medicine. Clin Sci (Lond) (2017) 131:1723-35. doi: 10.1042/CS20160253

3. Raundhal M, Morse C, Khare A, Oriss TB, Milosevic J, Trudeau J, et al. High IFN-Gamma and Low SLPI Mark Severe Asthma in Mice and Humans. JClin Invest (2015) 125:3037-50. doi: 10.1172/JCI80911

4. Ghoreschi K, Laurence A, O'Shea JJ. Janus Kinases in Immune Cell Signaling. Immunol Rev (2009) 228:273-87. doi: 10.1111/j.1600-065X. 2008.00754.x

5. O'Shea JJ, Schwartz DM, Villarino AV, Gadina M, McInnes IB, Laurence A. The JAK-STAT Pathway: Impact on Human Disease and Therapeutic Intervention. Annu Rev Med (2015) 66:311-28. doi: 10.1146/annurev-med051113-024537

6. Pernis AB, Rothman PB. JAK-STAT Signaling in Asthma. J Clin Invest (2002) 109:1279-83. doi: 10.1172/JCI0215786

7. Mesa RA. Ruxolitinib, a Selective JAK1 and JAK2 Inhibitor for the Treatment of Myeloproliferative Neoplasms and Psoriasis. IDrugs (2010) 13:394-403.

8. Gandhi VD, Davidson C, Asaduzzaman M, Nahirney D, Vliagoftis H. House Dust Mite Interactions With Airway Epithelium: Role in Allergic Airway Inflammation. Curr Allergy Asthma Rep (2013) 13:262-70. doi: 10.1007/ s11882-013-0349-9

9. Hashem T, Kammala AK, Thaxton K, Griffin RM, Mullany K, Panettieri RA Jr, et al. CD2 Regulates Pathogenesis of Asthma Induced by House Dust Mice Extract. Front Immunol (2020) 11:881. doi: 10.3389/fimmu.2020.00881

10. Lambrecht BN, Hammad H. The Immunology of Asthma. Nat Immunol (2015) 16:45-56. doi: 10.1038/ni.3049

11. Wu LC, Zarrin AA. The Production and Regulation of IgE by the Immune System. Nat Rev Immunol (2014) 14:247-59. doi: 10.1038/nri3632

12. Oliveria JP, Salter BM, Phan S, Obminski CD, Munoz CE, Smith SG, et al. Asthmatic Subjects With Allergy Have Elevated Levels of IgE+ B Cells in the Airways. J Allergy Clin Immunol (2017) 140:590-3.e599. doi: 10.1016/ j.jaci.2016.12.981

13. Oliveria JP, Salter BM, MacLean J, Kotwal S, Smith A, Harris JM, et al. Increased IgE(+) B Cells in Sputum, But Not Blood, Bone Marrow, or Tonsils, After Inhaled Allergen Challenge in Subjects With Asthma. Am J Respir Crit Care Med (2017) 196:107-9. doi: 10.1164/rccm.201611-2274LE

14. Gelfand EW, Joetham A, Wang M, Takeda K, Schedel M. Spectrum of TLymphocyte Activities Regulating Allergic Lung Inflammation. Immunol Rev (2017) 278:63-86. doi: 10.1111/imr.12561

15. Iwamura C, Nakayama T. Role of NKT Cells in Allergic Asthma. Curr Opin Immunol (2010) 22:807-13. doi: 10.1016/j.coi.2010.10.008

16. Wingett D, Nielson CP. Divergence in NK Cell and Cyclic AMP Regulation of T Cell CD40L Expression in Asthmatic Subjects. J Leukoc Biol (2003) 74:53141. doi: $10.1189 / \mathrm{jlb} .0303103$

17. Abdelaziz MH, Abdelwahab SF, Wan J, Cai W, Huixuan W, Jianjun C, et al. Alternatively Activated Macrophages; A Double-Edged Sword in Allergic Asthma. J Transl Med (2020) 18:58. doi: 10.1186/s12967-020-02251-w

18. Saradna A, Do DC, Kumar S, Fu QL, Gao P. Macrophage Polarization and Allergic Asthma. Transl Res (2018) 191:1-14. doi: 10.1016/j.trsl.2017.09.002

19. Oriss TB, Raundhal M, Morse C, Huff RE, Das S, Hannum R, et al. IRF5 Distinguishes Severe Asthma in Humans and Drives Th1 Phenotype and Airway Hyperreactivity in Mice. JCI Insight (2017) 2(10):e91019. doi: 10.1172/ jci.insight.91019

20. Zhu L, Ciaccio CE, Casale TB. Potential New Targets for Drug Development in Severe Asthma. World Allergy Organ J (2018) 11:30. doi: 10.1186/s40413018-0208-1

21. Palmqvist C, Wardlaw AJ, Bradding P. Chemokines and Their Receptors as Potential Targets for the Treatment of Asthma. Br J Pharmacol (2007) 151:725-36. doi: 10.1038/sj.bjp.0707263

22. Pease JE. Asthma, Allergy and Chemokines. Curr Drug Targets (2006) 7:3-12. doi: $10.2174 / 138945006775270204$

23. Gauthier M, Chakraborty K, Oriss TB, Raundhal M, Das S, Chen J, et al. Severe Asthma in Humans and Mouse Model Suggests a CXCL10 Signature Underlies Corticosteroid-Resistant Th1 Bias. JCI Insight (2017) 2(13):e94580. doi: $10.1172 /$ jci.insight. 94580
24. Fogli LK, Sundrud MS, Goel S, Bajwa S, Jensen K, Derudder E, et al. T CellDerived IL-17 Mediates Epithelial Changes in the Airway and Drives Pulmonary Neutrophilia. J Immunol (2013) 191:3100-11. doi: 10.4049/ jimmunol.1301360

25. Korn T, Bettelli E, Oukka M, Kuchroo VK. IL-17 and Th17 Cells. Annu Rev Immunol (2009) 27:485-517. doi: 10.1146/annurev.immunol.021908.132710

26. Kim RY, Pinkerton JW, Essilfie AT, Robertson AAB, Baines KJ, Brown AC, et al. Role for NLRP3 Inflammasome-Mediated, IL-1beta-Dependent Responses in Severe, Steroid-Resistant Asthma. Am J Respir Crit Care Med (2017) 196:283-97. doi: 10.1164/rccm.201609-1830OC

27. Ather JL, Ckless K, Martin R, Foley KL, Suratt BT, Boyson JE, et al. Serum Amyloid A Activates the NLRP3 Inflammasome and Promotes Th17 Allergic Asthma in Mice. J Immunol (2011) 187:64-73. doi: 10.4049/jimmunol. 1100500

28. Wadhwa R, Dua K, Adcock IM, Horvat JC, Kim RY, Hansbro PM. Cellular Mechanisms Underlying Steroid-Resistant Asthma. Eur Respir Rev (2019) 28:190096. doi: 10.1183/16000617.0096-2019

29. Oestreich KJ, Weinmann AS. Transcriptional Mechanisms That Regulate T Helper 1 Cell Differentiation. Curr Opin Immunol (2012) 24:191-5. doi: 10.1016/j.coi.2011.12.004

30. Wills-Karp M. Interleukin-13 in Asthma Pathogenesis. Immunol Rev (2004) 202:175-90. doi: 10.1111/j.0105-2896.2004.00215.x

31. Bonser LR, Erle DJ. Airway Mucus and Asthma: The Role of MUC5AC and MUC5B. J Clin Med (2017) 6:112. doi: 10.20944/preprints201711.0010.v1

32. Lu TX, Rothenberg ME. Diagnostic, Functional, and Therapeutic Roles of microRNA in Allergic Diseases. J Allergy Clin Immunol (2013) 132:3-13; quiz 14. doi: 10.1016/j.jaci.2013.04.039

33. Alhassan S, Hattab Y, Bajwa O, Bihler E, Singh AC. Asthma. Crit Care Nurs $Q$ (2016) 39:110-23. doi: 10.1097/CNQ.0000000000000104

34. Kudlacz E, Conklyn M, Andresen C, Whitney-Pickett C, Changelian P. The JAK-3 Inhibitor CP-690550 Is a Potent Anti-Inflammatory Agent in a Murine Model of Pulmonary Eosinophilia. Eur J Pharmacol (2008) 582:154-61. doi: 10.1016/j.ejphar.2007.12.024

35. Ashino S, Takeda K, Li H, Taylor V, Joetham A, Pine PR, et al. Janus Kinase 1/ 3 Signaling Pathways Are Key Initiators of TH2 Differentiation and Lung Allergic Responses. J Allergy Clin Immunol (2014) 133:1162-74. doi: 10.1016/ j.jaci.2013.10.036

36. Calama E, Ramis I, Domènech A, Carreño C, De Alba J, Prats N, et al. Tofacitinib Ameliorates Inflammation in a Rat Model of Airway Neutrophilia Induced by Inhaled LPS. Pulm Pharmacol Ther (2017) 43:60-7. doi: 10.1016/ j.pupt.2017.01.002

37. Winthrop KL. The Emerging Safety Profile of JAK Inhibitors in Rheumatic Disease. Nat Rev Rheumatol (2017) 13:234-43. doi: 10.1038/nrrheum. 2017.23

38. Ashcroft GS, Lei K, Jin W, Longenecker G, Kulkarni AB, Greenwell-Wild T, et al. Secretory Leukocyte Protease Inhibitor Mediates Non-Redundant Functions Necessary for Normal Wound Healing. Nat Med (2000) 6:114753. doi: $10.1038 / 80489$

39. Saito A, Horie M, Nagase T. TGF-Beta Signaling in Lung Health and Disease. Int J Mol Sci (2018) 19:2460. doi: 10.3390/ijms19082460

40. Michaeloudes C, Chang PJ, Petrou M, Chung KF. Transforming Growth Factor-Beta and Nuclear Factor E2-Related Factor 2 Regulate Antioxidant Responses in Airway Smooth Muscle Cells: Role in Asthma. Am J Respir Crit Care Med (2011) 184:894-903. doi: 10.1164/rccm.201011-17800C

41. Michaeloudes C, Sukkar MB, Khorasani NM, Bhavsar PK, Chung KF. TGFBeta Regulates Nox4, MnSOD and Catalase Expression, and IL-6 Release in Airway Smooth Muscle Cells. Am J Physiol Lung Cell Mol Physiol (2011) 300: L295-304. doi: 10.1152/ajplung.00134.2010

42. Brightling C, Berry M, Amrani Y. Targeting TNF-Alpha: A Novel Therapeutic Approach for Asthma. J Allergy Clin Immunol (2008) 121:5-10; quiz 11-12. doi: 10.1016/j.jaci.2007.10.028

43. Szabo SJ, Kim ST, Costa GL, Zhang X, Fathman CG, Glimcher LH. A Novel Transcription Factor, T-Bet, Directs Th1 Lineage Commitment. Cell (2000) 100:655-69. doi: 10.1016/S0092-8674(00)80702-3

44. Lametschwandtner G, Biedermann T, Schwärzler C, Günther C, Kund J, Fassl S, et al. Sustained T-Bet Expression Confers Polarized Human TH2 Cells With TH1-Like Cytokine Production and Migratory Capacities. J Allergy Clin Immunol (2004) 113:987-94. doi: 10.1016/j.jaci.2004.02.004 
45. Lighvani AA, Frucht DM, Jankovic D, Yamane H, Aliberti J, Hissong BD, et al. T-Bet Is Rapidly Induced by Interferon-Gamma in Lymphoid and Myeloid Cells. Proc Natl Acad Sci USA (2001) 98:15137-42. doi: 10.1073/ pnas. 261570598

46. Lugo-Villarino G, Maldonado-Lopez R, Possemato R, Penaranda C. \& Glimcher, L.H. T-Bet Is Required for Optimal Production of IFN-Gamma and Antigen-Specific T Cell Activation by Dendritic Cells. Proc Natl Acad Sci USA (2003) 100:7749-54. doi: 10.1073/pnas.133276710

47. Koya T, Miyahara N, Takeda K, Matsubara S, Matsuda H, Swasey C, et al. CD8+ T Cell-Mediated Airway Hyperresponsiveness and Inflammation Is Dependent on CD4+IL-4+ T Cells. J Immunol (2007) 179:2787-96. doi: 10.4049/jimmunol.179.5.2787

48. Schaller MA, Lundy SK, Huffnagle GB, Lukacs NW. CD8+ T Cell Contributions to Allergen Induced Pulmonary Inflammation and Airway Hyperreactivity. Eur J Immunol (2005) 35:2061-70. doi: 10.1002/ eji.200425715

49. Wills-Karp M. IL-12/IL-13 Axis in Allergic Asthma. J Allergy Clin Immunol (2001) 107:9-18. doi: 10.1067/mai.2001.112265

50. Philip R, Epstein LB. Tumour Necrosis Factor as Immunomodulator and Mediator of Monocyte Cytotoxicity Induced by Itself, Gamma-Interferon and Interleukin-1. Nature (1986) 323:86-9. doi: 10.1038/323086a0

51. Heine A, Held SA, Daecke SN, Wallner S, Yajnanarayana SP, Kurts C, et al. The JAK-Inhibitor Ruxolitinib Impairs Dendritic Cell Function In Vitro and In Vivo. Blood (2013) 122:1192-202. doi: 10.1182/blood-2013-03-484642

52. Kobayashi N, Nagumo H, Agematsu K. IL-10 Enhances B-Cell IgE Synthesis by Promoting Differentiation Into Plasma Cells, a Process That Is Inhibited by CD27/CD70 Interaction. Clin Exp Immunol (2002) 129:446-52. doi: 10.1046/ j.1365-2249.2002.01932.x

53. Jayaram L, Duong M, Pizzichini MM, Pizzichini E, Kamada D, Efthimiadis A, et al. Failure of Montelukast to Reduce Sputum Eosinophilia in High-Dose Corticosteroid-Dependent Asthma. Eur Respir J (2005) 25:41-6. doi: 10.1183/ 09031936.04.00008104

54. Lambrecht BN, Hammad H, Fahy JV. The Cytokines of Asthma. Immunity (2019) 50:975-91. doi: 10.1016/j.immuni.2019.03.018

55. Wills-Karp M, Luyimbazi J, Xu X, Schofield B, Neben TY, Karp CL, et al. Interleukin-13: Central Mediator of Allergic Asthma. Science (1998) 282:2258-61. doi: 10.1126/science.282.5397.2258

56. Chiaramonte MG, Mentink-Kane M, Jacobson BA, Cheever AW, Whitters MJ, Goad ME, et al. Regulation and Function of the Interleukin 13 Receptor Alpha 2 During a T Helper Cell Type 2-Dominant Immune Response. J Exp Med (2003) 197:687-701. doi: 10.1084/jem.20020903

57. Koya T, Takeda K, Kodama T, Miyahara N, Matsubara S, Balhorn A, et al. RANTES (CCL5) Regulates Airway Responsiveness After Repeated Allergen Challenge. Am J Respir Cell Mol Biol (2006) 35:147-54. doi: 10.1165/ rcmb.2005-0394OC

58. Isgrò M, Bianchetti L, Marini MA, Bellini A, Schmidt M, Mattoli S. The C-C Motif Chemokine Ligands CCL5, CCL11, and CCL24 Induce the Migration of Circulating Fibrocytes From Patients With Severe Asthma. Mucosal Immunol (2013) 6:718-27. doi: 10.1038/mi.2012.109

59. Besnard AG, Guillou N, Tschopp J, Erard F, Couillin I, Iwakura Y, et al. NLRP3 Inflammasome Is Required in Murine Asthma in the Absence of Aluminum Adjuvant. Allergy (2011) 66:1047-57. doi: 10.1111/j.13989995.2011.02586.x

60. Ray A, Kolls JK. Neutrophilic Inflammation in Asthma and Association With Disease Severity. Trends Immunol (2017) 38:942-54. doi: 10.1016/j.it.2017.07.003

61. Patel DF, Peiró T, Bruno N, Vuononvirta J, Akthar S, Puttur F, et al. Neutrophils Restrain Allergic Airway Inflammation by Limiting ILC2 Function and Monocyte-Dendritic Cell Antigen Presentation. Sci Immunol (2019) 4:eaax7006. doi: 10.1126/sciimmunol.aax7006

62. Li RF, Wang GF. JAK/STAT5 Signaling Pathway Inhibitor Ruxolitinib Reduces Airway Inflammation of Neutrophilic Asthma in Mice Model. Eur Rev Med Pharmacol Sci (2018) 22:835-43. doi: 10.26355/eurrev_ 201802_14320

63. Zhou H, Li J, Gao P, Wang Q, Zhang J. miR-155: A Novel Target in Allergic Asthma. Int J Mol Sci (2016) 17:1773. doi: 10.3390/ijms17101773

64. Lu C, Huang X, Zhang X, Roensch K, Cao Q, Nakayama KI, et al. miR-221 and miR-155 Regulate Human Dendritic Cell Development, Apoptosis, and
IL-12 Production Through Targeting of P27kip1, KPC1, and SOCS-1. Blood (2011) 117:4293-303. doi: 10.1182/blood-2010-12-322503

65. Malmhäll C, Alawieh S, Lu Y, Sjöstrand M, Bossios A, Eldh M, et al. MicroRNA-155 Is Essential for $\mathrm{T}(\mathrm{H}) 2$-Mediated Allergen-Induced Eosinophilic Inflammation in the Lung. J Allergy Clin Immunol (2014) 133:1429-38, 1438.e1421-7. doi: 10.1016/j.jaci.2013.11.008

66. Ceppi M, Pereira PM, Dunand-Sauthier I, Barras E, Reith W, Santos MA, et al. MicroRNA-155 Modulates the Interleukin-1 Signaling Pathway in Activated Human Monocyte-Derived Dendritic Cells. Proc Natl Acad Sci USA (2009) 106:2735-40. doi: 10.1073/pnas.0811073106

67. Banerjee A, Schambach F, DeJong CS, Hammond SM, Reiner SL. MicroRNA-155 Inhibits IFN-Gamma Signaling in CD4+ T Cells. Eur J Immunol (2010) 40:225-31. doi: 10.1002/eji.200939381

68. Rodriguez A, Vigorito E, Clare S, Warren MV, Couttet P, Soond DR, et al. Requirement of Bic/microRNA-155 for Normal Immune Function. Science (2007) 316:608-11. doi: 10.1126/science.1139253

69. Martinez-Nunez RT, Louafi F, Sanchez-Elsner T. The Interleukin 13 (IL-13) Pathway in Human Macrophages Is Modulated by microRNA-155 via Direct Targeting of Interleukin 13 Receptor Alpha1 (IL13Ralpha1). J Biol Chem (2011) 286:1786-94. doi: 10.1074/jbc.M110.169367

70. Chen M, Huang L, Zhang W, Shi J, Lin X, Lv Z, et al. MiR-23b Controls TGFBeta1 Induced Airway Smooth Muscle Cell Proliferation via TGFbetaR2/pSmad3 Signals. Mol Immunol (2016) 70:84-93. doi: 10.1016/j.molimm. 2015.12.012

71. Wang J, Wang HS, Su ZB. MicroRNA-142 Inhibits Proliferation and Promotes Apoptosis in Airway Smooth Muscle Cells During Airway Remodeling in Asthmatic Rats via the Inhibition of TGF-Beta -Dependent EGFR Signaling Pathway. Cell Physiol Biochem (2018) 47:1682-95. doi: $10.1159 / 000490986$

72. Zhang XY, Tang XY, Ma LJ, Guo YL, Li XS, Zhao LM, et al. Schisandrin B Down-Regulated lncRNA BCYRN1 Expression of Airway Smooth Muscle Cells by Improving miR-150 Expression to Inhibit the Proliferation and Migration of ASMC in Asthmatic Rats. Cell Prolif (2017) 50:e12382. doi: $10.1111 /$ cpr. 12382

73. Dileepan M, Jude JA, Rao SP, Walseth TF, Panettieri RA, Subramanian S, et al. MicroRNA-708 Regulates CD38 Expression Through Signaling Pathways JNK MAP Kinase and PTEN/AKT in Human Airway Smooth Muscle Cells. Respir Res (2014) 15:107. doi: 10.1186/s12931-014-0107-0

74. Cheng Z, Dai LL, Wang X, Jia LQ, Jing XG, Li PF, et al. MicroRNA-145 Down-Regulates Mucin 5AC to Alleviate Airway Remodeling and Targets EGFR to Inhibit Cytokine Expression. Oncotarget (2017) 8:46312-25. doi: 10.18632 /oncotarget.17933

75. Lu TX, Hartner J, Lim EJ, Fabry V, Mingler MK, Cole ET, et al. MicroRNA-21 Limits In Vivo Immune Response-Mediated Activation of the IL-12/IFNGamma Pathway, Th1 Polarization, and the Severity of Delayed-Type Hypersensitivity. J Immunol (2011) 187:3362-73. doi: 10.4049/jimmunol. 1101235

76. Lu TX, Munitz A, Rothenberg ME. MicroRNA-21 Is Up-Regulated in Allergic Airway Inflammation and Regulates IL-12p35 Expression. J Immunol (2009) 182:4994-5002. doi: 10.4049/jimmunol.0803560

77. Mattes J, Collison A, Plank M, Phipps S, Foster PS. Antagonism of microRNA-126 Suppresses the Effector Function of TH2 Cells and the Development of Allergic Airways Disease. Proc Natl Acad Sci USA (2009) 106:18704-9. doi: 10.1073/pnas.0905063106

78. Zhang K, Feng Y, Liang Y, Wu W, Chang C, Chen D, et al. Epithelial miR-206 Targets CD39/extracellular ATP to Upregulate Airway IL-25 and TSLP in Type 2-High Asthma. JCI Insight (2021) 6:e148103. doi: 10.1172/ jci.insight. 148103

79. Kumar M, Ahmad T, Sharma A, Mabalirajan U, Kulshreshtha A, Agrawal A, et al. Let-7 microRNA-Mediated Regulation of IL-13 and Allergic Airway Inflammation. J Allergy Clin Immunol (2011) 128:1077-85.e1-10. doi: 10.1016/ j.jaci.2011.04.034

80. Xiao C, Calado DP, Galler G, Thai TH, Patterson HC, Wang J, et al. MiR-150 Controls B Cell Differentiation by Targeting the Transcription Factor C-Myb. Cell (2016) 165:1027. doi: 10.1016/j.cell.2016.04.056

81. Zhu X, Wang X, Wang Y, Zhao Y. The Regulatory Network Among CircHIPK3, LncGAS5, and miR-495 Promotes Th2 Differentiation in 
Allergic Rhinitis. Cell Death Dis (2020) 11:216. doi: 10.1038/s41419-0202394-3

82. Xu W, Wang Y, Ma Y, Yang J. MiR-223 Plays a Protecting Role in Neutrophilic Asthmatic Mice Through the Inhibition of NLRP3 Inflammasome. Respir Res (2020) 21:116. doi: 10.1186/s12931-02001374-4

83. Johnnidis JB, Harris MH, Wheeler RT, Stehling-Sun S, Lam MH, Kirak O, et al. Regulation of Progenitor Cell Proliferation and Granulocyte Function by microRNA-223. Nature (2008) 451:1125-9. doi: 10.1038/ nature 06607

84. Keenan C, Nichols KE, Albeituni S. Use of the JAK Inhibitor Ruxolitinib in the Treatment of Hemophagocytic Lymphohistiocytosis. Front Immunol (2021) 12:614704. doi: 10.3389/fimmu.2021.614704

85. Calbet M, Ramis I, Calama E, Carreño C, Paris S, Maldonado M, et al. Novel Inhaled Pan-JAK Inhibitor, LAS194046, Reduces Allergen-Induced Airway Inflammation, Late Asthmatic Response, and pSTAT Activation in Brown Norway Rats. J Pharmacol Exp Ther (2019) 370:137-47. doi: 10.1124/ jpet.119.256263

86. Dengler HS, Wu X, Peng I, Rinderknecht CH, Kwon Y, Suto E, et al. LungRestricted Inhibition of Janus Kinase 1 Is Effective in Rodent Models of
Asthma. Sci Transl Med (2018) 10:eaao2151. doi: 10.1126/scitranslmed. aao2151

Conflict of Interest: The authors declare that the research was conducted in the absence of any commercial or financial relationships that could be construed as a potential conflict of interest.

Publisher's Note: All claims expressed in this article are solely those of the authors and do not necessarily represent those of their affiliated organizations, or those of the publisher, the editors and the reviewers. Any product that may be evaluated in this article, or claim that may be made by its manufacturer, is not guaranteed or endorsed by the publisher.

Copyright $(2021$ Subramanian, Hashem, Bahal, Kammala, Thaxton and Das. This is an open-access article distributed under the terms of the Creative Commons Attribution License (CC BY). The use, distribution or reproduction in other forums is permitted, provided the original author(s) and the copyright owner(s) are credited and that the original publication in this journal is cited, in accordance with accepted academic practice. No use, distribution or reproduction is permitted which does not comply with these terms. 